\title{
Fully Coupled Peridynamic Thermomechanics
}

Selda Oterkus and Erdogan Madenci*

Department of Aerospace and Mechanical Engineering, The University of Arizona, Tucson, AZ 85721, USA

Abigail Agwai

Intel Corporation, Chandler, AZ 85226, USA

\begin{abstract}
This study concerns the derivation of the coupled peridynamic (PD) thermomechanics equations based on thermodynamic considerations. The generalized peridynamic model for fully coupled thermomechanics is derived using the conservation of energy and the free-energy function. Subsequently, the bond-based coupled PD thermomechanics equations are obtained by reducing the generalized formulation. These equations are also cast into their nondimensional forms. After describing the numerical solution scheme, solutions to certain coupled thermomechanical problems with known previous solutions are presented.
\end{abstract}

\section{Introduction}

Thermomechanics concerns the influence of the thermal state of a solid body on the deformation and the influence of the deformation on the thermal state. In many cases, the effect of the deformation field on the thermal state may be ignored. This leads to a decoupled or uncoupled thermomechanical analysis, for which only the effect of the temperature field on the deformation is present. However, the uncoupled thermomechanics may not be satisfactory for certain transient problems. Experimental verification of the influence of the deformation on the thermal state exists (Thomson 1853, Stanley 2008). It was shown that an adiabatic solid experiences a temperature drop when it is strained in tension (Chadwick 1960, Fung 1965). Also, elastic bodies under tensile loading experience cooling below the yield stress; however, beyond the yield stress the bodies heat up due to the irreversible nature of plasticity (Nowinski 1978).

Also, the temperature field induced by structural loading may not be uniform. For example, when a beam with an initially uniform temperature is under bending, part of the beam is in tension while the other part is in compression. Due to the thermomechanical coupling, the part of the beam that is in tension cools and the region that is in compression heats up, establishing a thermal gradient. This leads to the onset of heat diffusion. The heat flow is irreversible; thus, some of the mechanical energy supplied to bend the beam is dissipated through its conversion to heat energy. This phenomenon is called thermoelastic damping and it plays a critical role in vibrations and wave propagation.

It is well known that during fracture in metals a plastic region, in which the material has locally yielded, occurs ahead of the crack tip. As a result, the mechanical energy is dissipated as heat and the temperature rises in the local region ahead of the crack tip. A 
slightly different phenomenon is observed for fracture in polymers. During fracture in polymers, it was experimentally observed that thermoelastic cooling is followed by a temperature rise due to the plastic zone and/or fracture process itself which exposes new surfaces (Rittel 1998). Consequently, in order to accurately model fracture, especially the crack tip, thermal consideration needs to be taken into account and a coupled thermomechanical analysis becomes necessary. The thermal and structural interaction becomes especially important for high-speed impact and penetration fracture problems (Brünig et al. 2011).

The derivation of the classical thermomechanics equation from a thermodynamic perspective did not occur till the mid 1950s (Biot 1956). Biot used generalized irreversible thermodynamics to formulate the classical thermomechanical laws in variational form, with the corresponding Euler equations representing the coupled momentum and energy equations.

The fully coupled thermomechanical equations based on the classical theory are well established. The classical equations of thermoelasticity are comprised of the deformation equation of motion with a thermoelastic constitutive law and the heat transfer equation with a structural (or deformational) heating and cooling term contributing to the thermal energy. For isotropic materials, the thermoelastic constitutive law includes the thermal stresses, which are related to the temperature gradient, while the structural heating and cooling are dependent on the thermal modulus and rate of dilatation. Depending on the structural idealization, the thermal modulus is defined as

$\beta_{c l}=(3 \lambda+2 \mu) \alpha=\frac{E \alpha}{1-2 v}$ for three dimensions ,

$\beta_{c l}=(2 \lambda+2 \mu) \alpha=\frac{E \alpha}{(1-v)}$ for two dimensions ,

$\beta_{c l}=(2 \mu) \alpha=E \alpha$ for one dimension,

in which $E$ is the elastic modulus, $\alpha$ is the coefficient of thermal expansion, and $v$ is the Poisson's ratio. The parameters $\lambda$ and $\mu$ are Lamé's constants.

Typically, the strength of coupling is measured via the nondimensional quantity known as the coupling coefficient and defined as

$\epsilon=\frac{\beta_{c l}{ }^{2} \Theta_{0}}{\rho c_{v}(\lambda+2 \mu)}$,

for which $\rho$ is the mass density, $c_{v}$ is the specific heat capacity, and $\Theta_{0}$ is the reference temperature at which the stress in the body is zero (Nowinski 1978). The presence of coupling makes the computational solution significantly complicated. If the coupling 
coefficient, Eq. (2) is small compared to unity, the presence of coupling may be disregarded. The coupling coefficients for metals are significantly lower than those of plastics. Steel, for example, has a coupling coefficient of about 0.011 while certain plastics have a value of $\epsilon=0.43$.

\section{Local Theory}

Various researchers analytically examined plane waves in thermoelastic solids (Chadwick and Sneddon 1958, Deresiewicz 1957). In a one-dimensional formulation, they showed that the presence of thermal and elastic waves, which are dispersed and attenuated. They also studied the effect of frequency on the phase velocity, attenuation, and damping. Later, Chadwick (1962) extended the analysis to two dimensions and investigated the propagation of thermoelastic waves in thin plates. Paria (1958) determined the temperature and stress distribution of a two-dimensional half-space problem using Laplace and Hankel transforms. Laplace transforms have also been used by Boley and Hetnarski (1968) to characterize propagating discontinuities in various onedimensional coupled thermoelastic problems. Fourier transforms were employed by Boley and Tolins (1962) to determine the mechanical and thermal response of a onedimensional semi-infinite bar with transient boundary conditions. The major challenge with transform methods is in finding the analytical inverse transforms - in many cases this is not possible and numerical inversion is necessary. Other analytical solution methods have been adopted to solve coupled thermoelastic problems. Soler and Brull (1965) used perturbation techniques and more recently Lychev et al. (2010) determined a closed-form solution by an expansion of the eigenfunctions generated by the coupled equations of motion and heat conduction.

Numerical approximations to the classical thermoelastic equations have been very commonly found using the finite element (FE) method. A transient thermoelastic FE model was developed by Nickell and Sackman (1968) and Ting and Chen (1982). The approximations from their FE model were compared against analytical solutions for various one-dimensional semi-infinite problems. Oden (1969) and Givoli and Rand (1995) developed dynamic thermoelastic FE models. Additionally, Chen and Weng (1988, 1989a, 1989b) modeled various thermoelastic problems such as the transient response of an axisymmetric infinite cylinder and an infinitely long plate using a finite element formulation in the Laplace transform domain. Hosseini-Tehrani and Eslami (2000) presented solutions for thermal and mechanical shocks in a finite domain based on the boundary element method (BEM) in conjunction with the Laplace-transform method in a time domain. They provided results for small time durations (early stages of the shock loads) using the numerical inversion of the Laplace-transform method.

Numerical solution schemes for thermomechanical problems are divided into two categories - monolithic schemes and staggered schemes. In monolithic schemes, the differential equations for different variables are solved simultaneously. On the other hand, for staggered or partitioned schemes, the solutions of the different variables are determined separately. In general, the staggered schemes have been favored over monolithic schemes, as the monolithic systems can be very large, making it unfeasible to solve practical problems. In addition, the mechanical and thermal parts of a 
thermomechanical problem may have very different time scales, hence requiring different time stepping schemes. However, the very nature of monolithic schemes renders this impossible.

One of the major issues associated with staggered numerical analysis of coupled thermomechanics is the concern of stability. When conditionally stable techniques are used to solve the coupled momentum and energy equations, a small time step size is required, which may be computationally impractical for certain problems. Even when various unconditionally stable methods are used to solve the equation of motion and heat transfer equation, the overall solution to the coupled problem may still be conditionally stable. A substantial amount of work has been done to combat this issue and to develop unconditionally stable staggered algorithms. Examples of such algorithms based on the finite element method include an adiabatic split scheme by Armero and Simo (1992) and various implicit-implicit and implicit-explicit schemes (Farhat et al. 1991, Liu and Zhang 1983, Liu and Chang 1985).

Although the solution to the local thermomechanical governing equations provides accurate deformation and temperature fields by employing the FE and BE methods, it presents singular stress and thermal flux in the presence of cracks due to undefined spatial derivatives of displacements and temperature at crack tips or along crack surfaces. When linear elastic fracture mechanics is adopted into the FE and BE analysis, special treatment is commonly needed in order to capture the correct singular behavior (mathematical artifact) at the crack tip. By using nonlocal theory, Eringen and Kim (1974a, b) showed that the stress field ahead of the crack tip is bounded as the crack tip is approached asymptotically, rather than unbounded as predicted by the classical continuum theory. The advantage of the nonlocal theory over the local theory in the area of fracture is made obvious by the fact that the nonlocal model predicts a physically meaningful finite stress field at the crack tip. Recently, Silling (2000) introduced a nonlocal theory that does not require spatial derivatives - the peridynamic (PD) theory. The governing field equations are valid regardless of the existence of a discontinuity. Furthermore, the PD theory permits crack initiation and growth through an internal failure parameter without modifying the computational thermal and deformation domains.

\section{Nonlocal Theory}

Research on nonlocal coupled thermomechanics is undoubtedly emerging. Classical nonlinear constitutive equations for nonlocal fully coupled thermoelasticity were presented by Huang (1999). Recently, Ardito and Comi (2009) have developed a fully nonlocal thermoelastic model that has an internal length scale. They analytically solved the nonlocal equations in order to determine the dissipation in microelectromechanical resonators. Comparison of their results with experimental observations revealed that the nonlocal model is able to capture the size effect that the local thermoelastic analysis is unable to capture. The work by Ardito and Comi (2009) illustrates the importance of nonlocality in small-scale problems. 
With the PD thermomechanical model, not only the problems that require nonlocality solvable, such as the microelectromechanical problems, but also the problems with discontinuities can be readily modeled. A crack that forms and propagates in a body with a varying temperature or temperature gradient is an example of such a problem. Therefore, the peridynamic approach to thermomechanics is advantageous as it not only accounts for nonlocality but also allows for coupled deformation and temperature fields to be determined in spite of cracks and other discontinuities. Uncoupled thermomechanics using the bond-based PD theory was developed by Kilic and Madenci (2010). However, no work has been published on fully coupled thermomechanics within the peridynamic framework.

\section{Peridynamic Thermomechanics}

Similar to the derivation of classical thermomechanical equations (Nowinski 1978), the generalized peridynamics for fully coupled thermomechanics is based on irreversible thermodynamics, i.e., the conservation of energy and the free energy density function.

\subsection{Peridynamic Thermal Diffusion with a Structural Coupling Term}

The first law of thermodynamics based on peridynamic quantities, accounting for the conservation of mechanical and thermal energy, has been given by Silling and Lehoucq (2010) as

$\dot{\varepsilon}=\underline{\mathbf{T}} \cdot \underline{\dot{\mathbf{Y}}}+\bar{Q}_{b}+q_{b}$,

where $\dot{\varepsilon}$ is the time rate of change of the internal energy storage density, and $q_{b}$ is the prescribed volumetric heat generation per unit mass. The term $\underline{\mathbf{T}} \cdot \underline{\dot{\mathbf{Y}}}$ represents the absorbed power density; it is the dot product of the force state and the time rate of change deformation state. The Appendix provides a brief description and properties of the concept of PD states. The absorbed power density in peridynamics is analogous to the stress power, $\boldsymbol{\sigma} \cdot \dot{\mathbf{F}}$ in classical continuum mechanics, where $\boldsymbol{\sigma}$ is the Piola stress tensor and $\mathbf{F}$ is the deformation gradient tensor. The variable $\bar{Q}$ is the rate of heat energy exchange with other material points, and it is given by

$$
\bar{Q}=\int_{H}\left(\underline{h}(\mathbf{x}, t)\left\langle\mathbf{x}^{\prime}-\mathbf{x}\right\rangle-\underline{h}\left(\mathbf{x}^{\prime}, t\right)\left\langle\mathbf{x}-\mathbf{x}^{\prime}\right\rangle\right) d V^{\prime},
$$

in which $\underline{h}$ is the heat flow scalar state. The domain of integration defined by $H$ includes the material points within its family or horizon, $\delta$. The quantity $\bar{Q}$ is related to

$\bar{Q}_{b}$ as $\bar{Q}=\rho \bar{Q}_{b}$.

The free energy density function is defined as (Silling and Lehoucq 2010)

$\psi=\varepsilon-\Theta £$ 
where $\Theta$ is the absolute temperature and $\mathscr{S}$ is the entropy density. The time derivative of Eq. (5) becomes

$\dot{\psi}=\dot{\varepsilon}-\dot{\Theta} \mathscr{S}-\Theta \dot{\mathcal{E}}$

Substituting for $\dot{\varepsilon}$ in Eq. (6) from the conservation of energy given in Eq. (3) leads to the following expression:

$\dot{\psi}=\underline{\mathbf{T}} \cdot \underline{\dot{\mathbf{Y}}}+\bar{Q}_{b}+q_{b}-\dot{\Theta} \mathscr{S}-\Theta \dot{\mathscr{S}}$.

The functional dependency of the free energy density and the entropy density can be defined in terms of the deformation state, time rate of change of the deformation state, and the temperature in the form

$\psi=\psi(\underline{\mathbf{Y}}, \underline{\dot{\mathbf{Y}}}, \Theta)$

$\mathscr{S}=\mathscr{S}(\underline{\mathbf{Y}}, \underline{\mathbf{Y}}, \Theta)$.

In conjunction with the chain rule, the time rate of change of the free energy density can be expressed as

$\dot{\psi}=\psi_{, \underline{\mathbf{Y}}} \cdot \dot{\mathbf{Y}}+\psi_{, \underline{\mathbf{Y}}} \cdot \underline{\mathbf{Y}}+\psi_{, \Theta} \dot{\Theta}$,

where the variable after the subscript comma indicates differentiation. If it is a state variable, its differentiation is known as the Fréchet derivative as explained in the Appendix.

Substituting from Eq. (7) into Eq. (9) results in

$\left(\Theta \dot{\mathcal{S}}-\bar{Q}_{b}-q_{b}\right)+\left(\psi_{, \Theta}+\mathscr{S}\right) \dot{\Theta}+\left(\psi_{, \underline{\mathbf{Y}}}-\underline{\mathbf{T}}\right) \cdot \underline{\dot{\mathbf{Y}}}+\psi_{, \underline{\dot{\mathbf{Y}}}} \cdot \underline{\ddot{\mathbf{Y}}}=0$

Adopting the assumption of Nowinski (1978) that $\underline{\dot{\mathbf{Y}}}, \underline{\ddot{\mathbf{Y}}}$, and $\dot{\Theta}$ vary independently, Eq. (10) leads to

$$
\begin{aligned}
& \Theta \dot{\mathcal{S}}-\bar{Q}_{b}-q_{b}=0, \\
& \mathscr{E}=-\psi_{, \Theta}, \\
& \underline{\mathbf{T}}=\psi_{, \underline{\mathbf{Y}}}, \\
& \psi_{, \underline{\mathbf{Y}}}=0 .
\end{aligned}
$$


where the first equation is only a special case of dissipation being equal to zero.

By using the free energy density, the first law of thermodynamics, and the ClausiusDuhem inequality, Silling and Lehoucq (2010), also determined these equations. (11a11d). In addition, they obtained expressions for the equilibrium, $\underline{\mathbf{T}}^{e}$, and dissipative, $\underline{\mathbf{T}}^{d}$, parts of force state vector as

$\underline{\mathbf{T}}^{e}(\underline{\mathbf{Y}}, \Theta)=\psi_{, \underline{\mathbf{Y}}}(\underline{\mathbf{Y}}, \Theta)$

$\underline{\mathbf{T}}^{d}(\underline{\mathbf{Y}}, \underline{\dot{\mathbf{Y}}}, \Theta) \cdot \underline{\dot{\mathbf{Y}}} \geq 0$

Using Eqs. (11b), (11d) and (8b) in conjunction with the chain rule, the time derivative of the entropy density may be rewritten in the form

$$
\dot{\mathscr{S}}=-\psi_{, \Theta \underline{\mathbf{Y}}} \cdot \dot{\mathbf{Y}}-\psi_{, \Theta \Theta} \dot{\Theta}
$$

Substituting from Eq. (13) into Eq. (11a) and multiplying by $\rho$ leads to

$$
\rho \Theta \psi_{, \Theta \underline{\mathbf{Y}}} \cdot \dot{\mathbf{Y}}+\rho \Theta \psi_{, \Theta \Theta} \dot{\Theta}+\bar{Q}+\rho q_{b}=0
$$

Based on the classical theory (Nowinski 1978), the specific heat capacity, $c_{v}$, can be related to the classical free energy density, $\bar{\psi}$, as

$$
\Theta \bar{\psi}_{, \Theta \Theta}=-c_{v} \text {. }
$$

The assumption of the classical free energy density at a point being equal to the peridynamic free energy density, $\psi$, leads to

$$
\Theta \psi_{, \Theta \Theta}=-c_{v} .
$$

Based on this observation, it is evident that the specific heat capacity has a similar meaning in the peridynamic theory as in the classical theory. Therefore, the term $\Theta \psi_{, \Theta \Theta}$ in Eq. (14) can be replaced by $-c_{v}$.

Based on the classical theory (Fung 1965), the thermal modulus $\beta_{i j}$ can be related to the classical free energy density $\bar{\psi}$ through

$$
\beta_{c l i j}=\rho \frac{\partial^{2} \bar{\psi}}{\partial e_{i j} \partial \Theta},
$$


where $e_{i j}$ is the strain tensor. Note that $\beta_{c l i j}=\beta_{c l} \delta_{i j}$ for isotropic materials.

Analogus to the thermal modulus of the classical theory, thermal modulus state, a vector state, $\underline{\mathbf{B}}$, can be defined as

$\underline{\mathbf{B}}=\rho \psi_{, \theta \underline{\mathbf{Y}}}$.

Substituting from Eqs. (4), (16), and (18) into Eq. (14) and after rearranging some of terms results in

$$
\rho c_{v} \dot{\Theta}(\mathbf{x}, t)=\int_{H}\left(\underline{h}(\mathbf{x}, t)\left\langle\mathbf{x}^{\prime}-\mathbf{x}\right\rangle-\underline{h}\left(\mathbf{x}^{\prime}, t\right)\left\langle\mathbf{x}-\mathbf{x}^{\prime}\right\rangle\right) d V^{\prime}+\Theta(\mathbf{x}, t) \underline{\mathbf{B}}(\mathbf{x}, t) \cdot \underline{\dot{Y}}(\mathbf{x}, t)+\rho q_{b}(\mathbf{x}, t)(19)
$$

Applying the definition of the vector state dot product (see Appendix) renders this equation

$$
\begin{aligned}
\rho c_{v} \dot{\Theta}(\mathbf{x}, t) & =\int_{H}\left(\left(\underline{h}(\mathbf{x}, t)\left\langle\mathbf{x}^{\prime}-\mathbf{x}\right\rangle-\underline{h}\left(\mathbf{x}^{\prime}, t\right)\left\langle\mathbf{x}-\mathbf{x}^{\prime}\right\rangle\right)+\Theta(\mathbf{x}, t) \underline{\mathbf{B}}\left\langle\mathbf{x}^{\prime}-\mathbf{x}\right\rangle \cdot \underline{\dot{Y}}\left\langle\mathbf{x}^{\prime}-\mathbf{x}\right\rangle\right) d V^{\prime} \\
& +\rho q_{b}(\mathbf{x}, t)
\end{aligned}
$$

in which the term $\underline{\mathbf{B}} \cdot \underline{\dot{\mathbf{Y}}}$ represents the effect of deformation on temperature. This implies that only the rate of change of extension influences the temperature; it is analogous to the classical heat conduction equation in which only the rate of dilatation appears. The final form of this equation can be obtained by defining $\underline{\dot{\mathbf{Y}}}$ and $\underline{\mathbf{B}}$ in terms of the time rate of change of the extension scalar state, $\underline{\dot{e}}$, and the thermal modulus scalar state, $\underline{\beta}$, as

$$
\begin{aligned}
& \underline{\mathbf{B}}\left\langle\mathbf{x}^{\prime}-\mathbf{x}\right\rangle=\underline{\beta}\left\langle\mathbf{x}^{\prime}-\mathbf{x}\right\rangle \frac{\mathbf{y}^{\prime}-\mathbf{y}}{\left|\mathbf{y}^{\prime}-\mathbf{y}\right|}, \\
& \underline{\mathbf{Y}}\left\langle\mathbf{x}^{\prime}-\mathbf{x}\right\rangle=\underline{\dot{e}}\left\langle\mathbf{x}^{\prime}-\mathbf{x}\right\rangle \frac{\mathbf{y}^{\prime}-\mathbf{y}}{\left|\mathbf{y}^{\prime}-\mathbf{y}\right|},
\end{aligned}
$$

in which the extension scalar state, $\underline{e}$, and thermal modulus scalar state, $\beta$, are defined as

$$
\begin{aligned}
& \underline{e}=\underline{y}-\underline{x}, \\
& \underline{\beta}=\rho \psi_{, \theta \underline{e}},
\end{aligned}
$$

with $\underline{y}=|\underline{\mathbf{Y}}|$ and $\underline{x}=|\underline{\mathbf{X}}|$. Thus, Eq. (20) can be recast as 


$$
\begin{aligned}
\rho c_{v} \dot{\Theta}(\mathbf{x}, t)= & \int_{H}\left(\left(\underline{h}(\mathbf{x}, t)\left\langle\mathbf{x}^{\prime}-\mathbf{x}\right\rangle-\underline{h}\left(\mathbf{x}^{\prime}, t\right)\left\langle\mathbf{x}-\mathbf{x}^{\prime}\right\rangle\right)+\Theta(\mathbf{x}, t) \underline{\beta}\left\langle\mathbf{x}^{\prime}-\mathbf{x}\right\rangle \underline{\dot{e}}\left\langle\mathbf{x}^{\prime}-\mathbf{x}\right\rangle\right) d V^{\prime} \\
& +\rho q_{b}(\mathbf{x}, t)
\end{aligned}
$$

\subsection{Peridynamic Deformation with a Thermal Coupling Term}

Based on the classical linear theory of thermoelasticity (Nowinski 1978), the free energy density is a potential function given by

$\bar{\psi}=\bar{\psi}\left(e_{i j}, T\right)=\frac{1}{2} c_{i j k l} e_{i j} e_{k l}-\beta_{c l i j} e_{i j} T-\frac{c_{v}}{2 \Theta_{0}} T^{2}$,

where $c_{i j k l}$ is the elastic moduli of the material, $T=\Theta-\Theta_{0}$, and $\Theta_{0}$ is the reference temperature. A similar approach is adopted herein for the derivation of the peridynamic deformation equation with a thermal coupling term.

Silling (2009) developed a linearized form of the state-based peridynamics for small elastic deformation by introducing the force vector state, $\underline{\mathbf{T}}$, as

$\underline{\mathbf{T}}=\underline{\mathbf{T}}(\underline{\mathbf{U}})$,

where $\underline{\mathbf{U}}$ is the displacement vector state. The free energy density function is expressed in terms of $\underline{\mathbf{U}}$ as

$\psi(\underline{\mathbf{U}})=\psi\left(\underline{\mathbf{Y}}^{0}\right)+\underline{\mathbf{T}}^{0} \cdot \underline{\mathbf{U}}+\frac{1}{2} \underline{\mathbf{U}} \cdot \underline{\mathbb{K}} \cdot \underline{\mathbf{U}}$,

where $\underline{\mathbf{Y}}^{0}$ and $\underline{\mathbf{T}}^{0}$ are defined as the equilibrated deformation and force states, respectively. The double state $\mathbb{K}$ is called the modulus state, and it is given by Silling (2009) as

$\underline{\mathbb{K}}=\underline{\mathbf{T}}_{, \underline{\mathbf{Y}}}^{0}$.

For linear thermoelastic material response, in accordance with Eq. (23), this form of the free energy is modified by including $T$ and $\underline{\mathbf{U}}$ as

$\psi(\underline{\mathbf{U}}, T)=\psi\left(\underline{\mathbf{Y}}^{0}\right)+\underline{\mathbf{T}}^{0} \cdot \underline{\mathbf{U}}+\frac{1}{2} \underline{\mathbf{U}} \cdot \underline{\mathbb{K}} \cdot \underline{\mathbf{U}}-\underline{\mathbf{B}} \cdot \underline{\mathbf{U}} T-\frac{c_{v}}{2 \Theta_{0}} T^{2}$

Invoking this equation into Eq. (11c) results in the explicit form of the force state as

$\underline{\mathbf{T}}=\underline{\mathbb{K}} \cdot \underline{\mathbf{U}}-\underline{\mathbf{B}} T$ 
It represents the state-based constitutive relation for a linearized peridynamic thermoelastic material. Substituting from Eq. (28) into the peridynamic equation of motion derived by Silling et al. (2007) results in the following:

$$
\rho \ddot{\mathbf{u}}(\mathbf{x}, t)=\int_{H}\left[(\underline{\mathbb{K}} \cdot \underline{\mathbf{U}}-\underline{\mathbf{B}} T)(\mathbf{x}, t)\left\langle\mathbf{x}^{\prime}-\mathbf{x}\right\rangle-(\underline{\mathbb{K}} \cdot \underline{\mathbf{U}}-\underline{\mathbf{B}} T)\left(\mathbf{x}^{\prime}, t\right)\left\langle\mathbf{x}-\mathbf{x}^{\prime}\right\rangle\right] d V^{\prime}+\mathbf{b}(\mathbf{x}, t)
$$

in which the term $\underline{\mathbf{B}}\left\langle\mathbf{x}^{\prime}-\mathbf{x}\right\rangle T$ represents the effect of the thermal state on deformation. For a nonlinear elastic material model, the free energy is composed of a thermal and a mechanical component. Therefore, one possible form of the force state can be

$\underline{\mathbf{T}}=\underline{\nabla} W-\underline{\mathbf{B}} T$,

in which $W$ is the deformational strain energy density and $\underline{\nabla} W$ is its Fréchet derivative. The part of the force state, $\underline{\mathbf{T}}_{s}$, that includes only the structural deformation can be defined as

$\underline{\mathbf{T}}_{s}=\underline{\nabla} W$.

Substituting from these equations into the peridynamic equation of motion derived by Silling et al. (2007) can be recast as

$$
\rho \ddot{\mathbf{u}}(\mathbf{x}, t)=\int_{H}\left[\left(\underline{\mathbf{T}}_{s}\left\langle\mathbf{x}^{\prime}-\mathbf{x}\right\rangle-\underline{\mathbf{B}}\left\langle\mathbf{x}^{\prime}-\mathbf{x}\right\rangle T\right)-\left(\underline{\mathbf{T}}_{s}^{\prime}\left\langle\mathbf{x}-\mathbf{x}^{\prime}\right\rangle-\underline{\mathbf{B}}^{\prime}\left\langle\mathbf{x}-\mathbf{x}^{\prime}\right\rangle T^{\prime}\right)\right] d V^{\prime}+\mathbf{b}(\mathbf{x}, t)
$$

where $\underline{\mathbf{T}}_{s}=\underline{\mathbf{T}}_{s}(\mathbf{x}, t)$ and $\underline{\mathbf{T}}_{s}^{\prime}=\underline{\mathbf{T}}_{s}\left(\mathbf{x}^{\prime}, t\right) ;$ similar notation is used for $\underline{\mathbf{B}}$ and $T$.

\subsection{Bond - Based Peridynamic Thermomechanics}

The general forms of ordinary state-based PD heat transfer equation and equation of motion are given by Eqs. (22) and (32). When the interactions between material points are only pairwise, the equation of motion reduces to the bond-based PD as derived by Kilic and Madenci (2010) in the form

$$
\begin{aligned}
\rho \ddot{\mathbf{u}}(\mathbf{x}, t)= & \int_{H}\left\{\left(\frac{c}{2} \frac{\left|\mathbf{y}^{\prime}-\mathbf{y}\right|-\left|\mathbf{x}^{\prime}-\mathbf{x}\right|}{\left|\mathbf{x}^{\prime}-\mathbf{x}\right|}-\frac{c}{2} \alpha T\right) \frac{\mathbf{y}^{\prime}-\mathbf{y}}{\left|\mathbf{y}^{\prime}-\mathbf{y}\right|}\right. \\
& \left.-\left(\frac{c}{2} \frac{\left|\mathbf{y}-\mathbf{y}^{\prime}\right|-\left|\mathbf{x}-\mathbf{x}^{\prime}\right|}{\left|\mathbf{x}-\mathbf{x}^{\prime}\right|}-\frac{c}{2} \alpha T^{\prime}\right) \frac{\mathbf{y}-\mathbf{y}^{\prime}}{\left|\mathbf{y}-\mathbf{y}^{\prime}\right|}\right\} d V^{\prime}+\mathbf{b}(\mathbf{x}, t) .
\end{aligned}
$$

where $c$ is referred to as the "bond constant" by Silling and Askari (2005). For an isotropic material, this bond constant can be expressed in term of Young's Modulus, $E$ and horizon size, $\delta$. Comparison of this equation with Eq. (32) leads to the explicit forms of 
$\underline{\mathbf{T}}_{s}\left\langle\mathbf{x}^{\prime}-\mathbf{x}\right\rangle=\frac{c}{2} \frac{\left|\mathbf{y}^{\prime}-\mathbf{y}\right|-\left|\mathbf{x}^{\prime}-\mathbf{x}\right|}{\left|\mathbf{x}^{\prime}-\mathbf{x}\right|} \frac{\mathbf{y}^{\prime}-\mathbf{y}}{\left|\mathbf{y}^{\prime}-\mathbf{y}\right|}$

and

$\underline{\mathbf{B}}\left\langle\mathbf{x}^{\prime}-\mathbf{x}\right\rangle=\frac{c}{2} \alpha \frac{\mathbf{y}^{\prime}-\mathbf{y}}{\left|\mathbf{y}^{\prime}-\mathbf{y}\right|}$

Comparison of Eq. (34b) with Eq. (21a) results in the expression for thermal modulus scalar state $\underline{\beta}$ as

$\underline{\beta}\left\langle\mathbf{x}^{\prime}-\mathbf{x}\right\rangle=\frac{c}{2} \alpha$

The difference between the generalized heat transfer equation, and thermomechanical heat transfer equation for an isotropic material, Eq. (22), is due to the deformational heating and cooling term, $(\beta \underline{\dot{e}})$. Therefore, the bond-based coupled thermomechanical heat transfer equation for isotropic materials can be cast as

$\rho c_{v} \dot{\Theta}(\mathbf{x}, t)=\int_{H}\left(f_{h}-\Theta \frac{c}{2} \alpha \dot{e}\right) d V^{\prime}+\rho q_{b}(\mathbf{x}, t)$

where $f_{h}$ is the thermal response function, and $\dot{e}$ is the time rate of change of the extension between the material points. This extension is defined as

$e=|\boldsymbol{\eta}+\xi|-|\xi|$,

with its time rate of change

$\dot{e}=\frac{\boldsymbol{\eta}+\xi}{|\boldsymbol{\eta}+\xi|} \cdot \dot{\boldsymbol{\eta}}$

where $\dot{\boldsymbol{\eta}}$ is the time rate of change of the relative displacement vector. Equation (36) can be rewritten in terms of the change in temperature, $T=\Theta-\Theta_{0}$, by replacing $\Theta$ with $T+\Theta_{0}$ and $\dot{\Theta}$ with $\dot{T}$ as

$\rho c_{v} \dot{T}(\mathbf{x}, t)=\int_{H}\left(f_{h}-\left(T+\Theta_{o}\right) \frac{c}{2} \alpha \dot{e}\right) d V^{\prime}+\rho q_{b}(\mathbf{x}, t)$

or 


$$
\rho c_{v} \dot{T}(\mathbf{x}, t)=\int_{H}\left(f_{h}-\Theta_{o}\left(\frac{T}{\Theta_{o}}+1\right) \frac{c}{2} \alpha \dot{e}\right) d V^{\prime}+\rho q_{b}(\mathbf{x}, t) .
$$

As suggested by Nowinski (1978), if the temperature change, $T$, is very small when compared with the reference temperature, $\Theta_{o}$, Eq. (38b) can be approximated as

$$
\rho c_{v} \dot{T}(\mathbf{x}, t)=\int_{H}\left(f_{h}-\Theta_{o} \frac{c}{2} \alpha \dot{e}\right) d V^{\prime}+\rho q_{b}(\mathbf{x}, t)
$$

Note that for the uncoupled case, this equation reduces to the PD heat conduction equation as given by Bobaru and Duangpanya (2010, 2012). Substituting for the thermal response (heat flow density) function, $f_{h}$ as suggested by Agwai (2011) leads to its final form as

$$
\rho c_{v} \dot{T}(\mathbf{x}, t)=\int_{H}\left(\kappa \frac{\tau}{|\xi|}-\Theta_{o} \frac{c}{2} \alpha \dot{e}\right) d V^{\prime}+\rho q_{b}(\mathbf{x}, t) .
$$

in which $\tau=T\left(\mathbf{x}^{\prime}\right)-T(\mathbf{x})$ and $|\xi|=\left|\mathbf{x}^{\prime}-\mathbf{x}\right|$ represent the difference in temperature and distance between the material points, respectively. The microconductivity, $\kappa$ is a peridynamic material parameter that can be expressed in terms of thermal conductivity, $k$. It can be obtained by equating the PD thermal potential to the classical thermal potential at a point as explained by Agwai (2011).

From Eq. (33), the bond-based PD equation of motion including the effect of temperature can be rewritten as

$$
\rho \ddot{\mathbf{u}}(\mathbf{x}, t)=\int_{H} \frac{\xi+\boldsymbol{\eta}}{|\xi+\boldsymbol{\eta}|}\left(c s-c \alpha T_{\text {avg }}\right) d V^{\prime}+\mathbf{b}(\mathbf{x}, t),
$$

in which $c$ is the peridynamic material parameter. The initial relative position and relative displacement vectors are defined as $\xi=\mathbf{x}^{\prime}-\mathbf{x}$ and $\boldsymbol{\eta}=\mathbf{u}^{\prime}-\mathbf{u}$, respectively. The parameter $s$ represents the stretch between material points $\mathbf{x}^{\prime}$ and $\mathbf{x}$, and $T_{\text {avg }}$ is the mean value of the change in temperatures at material points $\mathbf{x}^{\prime}$ and $\mathbf{x}$ defined as

$$
T_{\text {avg }}=\frac{T+T^{\prime}}{2}
$$

Introducing $\beta$ as the bond-based peridynamic thermal modulus, the final form of the fully coupled bond-based thermomechanical equations for isotropic materials become 
$\rho c_{v} \dot{T}(\mathbf{x}, t)=\int_{H}\left(\kappa \frac{\tau}{|\xi|}-\Theta_{o} \frac{\beta}{2} \dot{e}\right) d V^{\prime}+h_{q}(\mathbf{x}, t)$

with $h_{q}=\rho q_{b}$ representing the heat source due to volumetric heat generation, and

$\rho \ddot{\mathbf{u}}(\mathbf{x}, t)=\int_{H} \frac{\xi+\boldsymbol{\eta}}{|\xi+\boldsymbol{\eta}|}\left(c s-\beta T_{a v g}\right) d V^{\prime}+\mathbf{b}(\mathbf{x}, t)$,

with

$\beta=c \alpha$

The first equation is the conservation of thermal energy (i.e., the heat transfer equation) with a contribution from deformational heating and cooling, and the second equation is the conservation of linear momentum (i.e., the equation of motion) with a thermoelastic constitutive relation.

\section{Nondimensional Form of Thermomechanical Equations}

The nondimensional form of an equation or system of equations involves eliminating the units associated with the variables and parameters. For coupled systems, various parameters may differ in size and the effects of certain parameters may not be apparent. The nondimensional form of equations can permit the effects of the different parameters to become more evident. The appropriate scaling, relative measure of quantities, and characteristic properties of the system, such as time constants, length scales, and resonance frequencies, can be revealed through nondimensionalization.

\subsection{Characteristic Length and Time Scales}

The characteristic length/time quantity for heat conduction is the diffusivity defined as

$\gamma=\frac{k}{\rho c_{v}}=\frac{\ell^{* 2}}{t^{*}}$

where $\ell^{*}$ and $t^{*}$ represent the characteristic length and time, respectively. For the equation of motion, the characteristic length/time is the elastic wave speed. The square of the elastic wave speed, $\tilde{a}$, is

$\tilde{a}^{2}=\frac{(\lambda+2 \mu)}{\rho}=\frac{\ell^{* 2}}{t^{* 2}}$,

where $\lambda$ and $\mu$ are Lamé's constants. Combining the characteristic length/time scale from Eqs. (44) and (45) leads to the characteristic length and time for thermomechanics as 
$\ell^{*}=\frac{\gamma}{\tilde{a}} \quad$ and $\quad t^{*}=\frac{\gamma}{\tilde{a}^{2}}$

The characteristic length and time are typically employed in the nondimensionalization of the thermomechanical equations.

\subsection{Nondimensional Parameters}

The nondimensional form of Eq. (43) can be achieved by adopting the approach by Nickell and Sackman (1968) using Eqs. (44) and (45) for thermal diffusivity and the square of the elastic wave speed. The nondimensional variables are denoted with an overscore. The nondimensionalization procedure for length related variables, i.e., $x, \delta, A$, and $V$ (the volume), employs the characteristic length, and they are defined as

$x=\left(\frac{\gamma}{\tilde{a}}\right) \bar{x}, \quad \delta=\left(\frac{\gamma}{\tilde{a}}\right) \bar{\delta}, \quad A=\left(\frac{\gamma}{\tilde{a}}\right)^{2} \bar{A}$ and $V=\left(\frac{\gamma}{\tilde{a}}\right)^{3} \bar{V}$

The displacement is nondimensionalized as

$u=\left(\frac{\gamma}{\tilde{a}}\right) \frac{\beta_{c l} \Theta_{o}}{(\lambda+2 \mu)} \bar{u}$

The stretch is nondimensionalized as

$s=\frac{\beta_{c l} \Theta_{o}}{(\lambda+2 \mu)} \bar{s}$

The time is scaled using the characteristic length as

$t=\left(\frac{\gamma}{\tilde{a}^{2}}\right) \bar{t}$

The nondimensionalization for the velocity-related variables are achieved by

$v=\frac{\beta_{c l} \Theta_{o}}{(\lambda+2 \mu)} \tilde{a} \bar{v} \quad$ and $\quad \dot{e}=\frac{\beta_{c l} \Theta_{o}}{(\lambda+2 \mu)} \tilde{a} \bar{e}$

Finally, the temperature and temperature difference are nondimensionalized as

$T=\Theta_{o} \bar{T} \quad$ and $\quad \tau=\Theta_{o} \bar{\tau}$.

It is worth noting that the definitions of thermal modulus, bulk modulus, Lamé constants, shear modulus, peridynamic parameters, and microconductivity depend on the structural 
idealization. Their definitions for one-, two-, and three-dimensional analysis are summarized as:

One-dimensional analysis

$\lambda=0, \mu=\frac{E}{2}, \alpha=\frac{\beta_{c l}}{2 \mu}, c=\frac{2 E}{A \delta^{2}}, \kappa=\frac{2 k}{A \delta^{2}}$.

Two-dimensional analysis

$\lambda=\frac{E v}{(1-v)(1+v)}, \mu=\frac{E}{2(1+v)}, \alpha=\frac{\beta_{c l}}{2 \lambda+2 \mu}, c=\frac{9 E}{\pi h \delta^{3}}, \kappa=\frac{6 k}{\pi h \delta^{3}}$.

Three-dimensional analysis

$\lambda=\frac{E v}{(1+v)(1-2 v)}, \mu=\frac{E}{2(1+v)}, \alpha=\frac{\beta_{c l}}{3 \lambda+2 \mu}, c=\frac{12 E}{\pi \delta^{4}}, \kappa=\frac{6 k}{\pi \delta^{4}}$.

Equating the coefficient of thermal expansion from Eqs. (1) and (43c) leads to the thermal modulus as

$\beta=\frac{\beta_{c l}}{3 \lambda+2 \mu} c$ for three dimensions ,

$\beta=\frac{\beta_{c l}}{2 \lambda+2 \mu} c$ for two dimensions,

$\beta=\frac{\beta_{c l}}{2 \mu} c$ for one dimension

Substituting from Eqs. (47) to (52) with the dimensional considerations from Eq. (53), the fully coupled bond-based thermomechanical equations in the absence of body force and heat source can be cast into their nondimensional forms:

One-dimensional analysis

$\frac{\partial^{2} \overline{\mathbf{u}}}{\partial \bar{t}^{2}}=\frac{2}{\bar{\delta}^{2} \bar{A}} \int_{H} \frac{\xi+\boldsymbol{\eta}}{|\xi+\boldsymbol{\eta}|}\left(\bar{s}-\bar{T}_{a v g}\right) d \bar{V}_{\mathbf{x}^{\prime}}+\overline{\mathbf{b}}$

$\frac{\partial \bar{T}}{\partial \bar{t}}=\frac{2}{\bar{\delta}^{2} \bar{A}} \int_{H}\left(\frac{\bar{\tau}}{|\bar{\xi}|}-\epsilon \frac{\overline{\dot{e}}}{2}\right) d \bar{V}_{\mathbf{x}^{\prime}}+\bar{h}_{s}$

Two-dimensional analysis

$\frac{\partial^{2} \overline{\mathbf{u}}}{\partial \bar{t}^{2}}=\frac{9(1-v)}{\pi \bar{\delta}^{3} \bar{h}} \int_{H} \frac{\xi+\boldsymbol{\eta}}{|\xi+\boldsymbol{\eta}|}\left((1+v) \bar{s}-\bar{T}_{a v g}\right) d \bar{V}_{\mathbf{x}^{\prime}}+\overline{\mathbf{b}}$, 


$$
\frac{\partial \bar{T}}{\partial \bar{t}}=\frac{6}{\pi \bar{\delta}^{3} \bar{h}} \int_{H}\left(\frac{\bar{\tau}}{|\bar{\xi}|}-\frac{3}{4}(1-v) \epsilon \overline{\dot{e}}\right) d \bar{V}_{\mathbf{x}^{\prime}}+\bar{h}_{q}
$$

Three-dimensional analysis

$$
\begin{aligned}
& \frac{\partial^{2} \overline{\mathbf{u}}}{\partial \bar{t}^{2}}=\frac{6}{\pi \bar{\delta}^{4}} \int_{H} \frac{\xi+\boldsymbol{\eta}}{|\xi+\boldsymbol{\eta}|}\left(\frac{1+v}{1-v} \bar{s}-\bar{T}_{a v g}\right) d \bar{V}_{\mathbf{x}^{\prime}}+\overline{\mathbf{b}} \\
& \frac{\partial \bar{T}}{\partial \bar{t}}=\frac{6}{\pi \bar{\delta}^{4}} \int_{H}\left(\frac{\bar{\tau}}{|\bar{\xi}|}-\frac{1}{2} \epsilon \overline{\dot{e}}\right) d \bar{V}_{\mathbf{x}^{\prime}}+\bar{h}_{q},
\end{aligned}
$$

in which the nondimensional coupling coefficient, $\epsilon$, body force density, $\overline{\mathbf{b}}$, and heat source due to volumetric heat generation, $\bar{h}_{q}$, are defined as

$$
\begin{gathered}
\epsilon=\frac{\beta_{c l}^{2} \Theta_{0}}{\rho c_{v}(\lambda+2 \mu)}, \\
\overline{\mathbf{b}}=\frac{\gamma(\lambda+2 \mu)}{\rho \tilde{a}^{3} \beta_{c l} \Theta_{0}} \mathbf{b}, \\
\bar{h}_{q}=\frac{\gamma}{\rho c_{v} \tilde{a}^{2} \Theta_{0}} h_{q} .
\end{gathered}
$$

The coupling coefficient $\epsilon$ measures the strength of thermal and deformation coupling and it appears in the nondimensional thermomechanical equations associated with the heating and cooling term due to deformation. The coupling coefficient is singled out of the nondimensional form of these peridynamic equations in a similar manner as it is singled out of the classical thermomechanical equations, as illustrated by Nickell and Sackmann (1968). The nondimensional equation represents decoupled thermomechanics for $\epsilon=0$. It is worth noting that the equation of motion still contains the effect of temperature even if $\epsilon=0$.

\section{Boundary conditions}

The resulting PD equations do not contain any spatial derivatives; thus, constraint conditions are, in general, not necessary for the solution of an integro-differential equations. . However, such conditions can be imposed by prescribing constraints on displacement and temperature fields in a "fictitious material layer" along the boundary of a nonzero volume. Based on numerical experiments, Macek and Silling (2007) suggested that the extent of the fictitious boundary layer be equal to the horizon, $\delta$, in order to ensure the imposed prescribed constraints are sufficiently reflected on the actual material region. 


\subsection{Temperature and flux constraints}

As shown in Fig. 1, the prescribed boundary temperature, $\bar{T}_{b}$ can be imposed in a layer of fictitious region as

$\bar{T}_{f}\left(\mathbf{x}_{f}, t+\Delta t\right)=2 \bar{T}_{b}\left(\mathbf{x}_{b}, t+\Delta t\right)-\bar{T}(\mathbf{x}, t)$

where $\bar{T}_{f}$ and $\mathbf{x}_{f}$ represent the temperature and material points in the fictitious region, $\mathcal{R}_{t}$. In the case of $\bar{T}_{b}=0$, this representation enforces the temperature variation in the fictitious region to become the negative mirror image of the temperature variation near the boundary surface in the actual material.

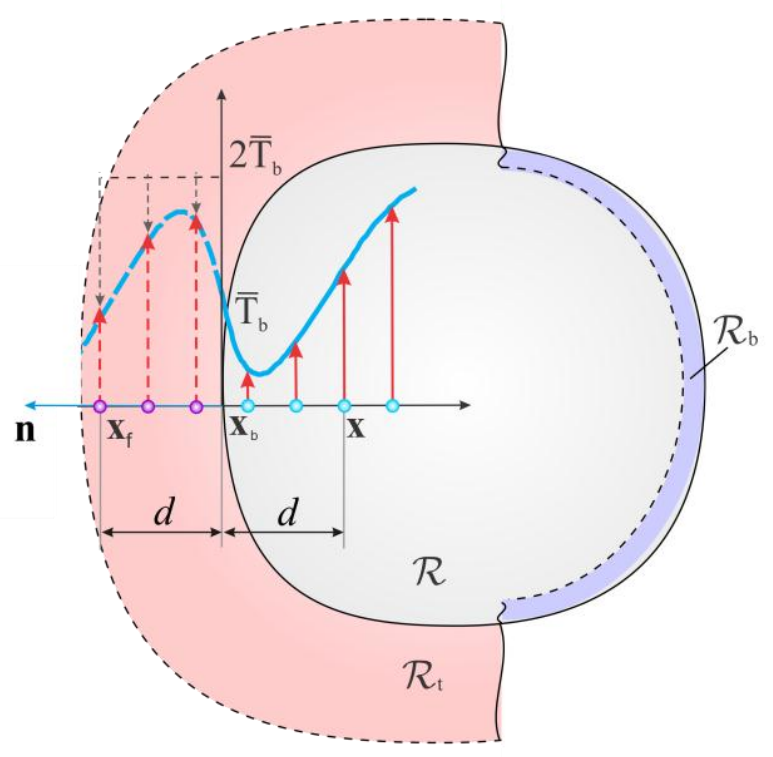

Figure 1. Imposing temperature and flux constraints

Application of flux type of boundary condition is accomplished by first calculating the rate of heat entering through the bounding surface then converting the heat flow rate, $\dot{Q}$ to a heat generation per unit volume and then specifying this volumetric heat generation to material points in the boundary region, $\mathcal{R}_{b}$. Assuming the cross sectional area is constant for each material point, conversion is achieved by

$\tilde{Q}=\frac{\dot{Q}}{V_{f}}=\frac{-\int_{\mathcal{S}_{f}} \mathbf{q} \cdot \mathbf{n} d \mathcal{S}}{V_{f}}=-\frac{\mathbf{q} \cdot \mathbf{n} \mathcal{S}_{f}}{\mathcal{S}_{f} \Delta}=-\frac{\mathbf{q} \cdot \mathbf{n}}{\Delta}$ 
where $\tilde{Q}$ is the volumetric heat generation, $\mathbf{q}$ is the heat flux, $\mathcal{S}_{f}$ is the area over which the heat flux is applied, $\Delta$ depth (spacing between material points) and $V_{f}$ is the volume of the boundary region.

If there exists no specified flux, $\mathbf{q}^{*}(\mathbf{x}, t)=0$, volumetric heat generation, $\tilde{Q}$ calculated from Eq. (60) vanishes. Thus, the implementation of zero flux boundary condition can be viewed as imposing a zero valued volumetric heat generation. Alternative to this implementation, zero flux can be achieved by assigning the mirror image of the temperature values near the boundary in the actual domain to the material points in the fictitious region as

$\bar{T}_{f}\left(\mathbf{x}_{f}, t+\Delta t\right)=\bar{T}(\mathbf{x}, t)$

\subsection{Displacement/velocity and pressure constraints}

The prescribed displacement or velocity vector $\overline{\mathbf{U}}_{b}$ and $\overline{\mathbf{V}}(t)$ can be imposed through the material points in the fictitious material layer.

$\overline{\mathbf{u}}(\mathbf{x}, t)=\overline{\mathbf{U}}_{b}$

and

$\dot{\overline{\mathbf{u}}}(\mathbf{x}, t)=\overline{\mathbf{V}}(t)$

The distributed non-dimensional pressure $\bar{p}(\mathbf{x}, t)$ is imposed in the form of body force density on the material points in the boundary region as

$\overline{\mathbf{b}}(\mathbf{x}, t)=-\frac{1}{\Delta} \bar{p}(\mathbf{x}, t) \mathbf{n}$

in which $\mathbf{n}$ is unit normal to the boundary.

\section{Numerical Procedure}

For numerically approximating the solution to the classical fully coupled equations for thermoelasticity, one of two different time stepping strategies is generally employed by researchers. The monolithic or simultaneous scheme is one time stepping strategy. For a monolithic algorithm, the time stepping scheme is applied simultaneously to the full system of equations and the unknown variables are solved for at the same time. If the time stepping scheme for the monolithic algorithm is implicit, unconditional stability is usually achieved. However, monolithic algorithms can result in practically large systems, in spite of their unconditional stability. For the staggered or partitioned scheme, the coupled system of equations are split, typically according to two different fields, the displacement and temperature fields. Each field is then individually treated with a different time stepping algorithm. Staggered algorithms generally circumvent the 
shortcomings of their monolithic counterparts; however, this is often accomplished at the expense of the unconditional stability. In many scenarios, even when unconditionally stable time stepping schemes are used to solve each partitioned equation, the overall stability of the thermomechanical system of equations is only conditional (Wood 1990). As a result, a good deal of work has been performed to successfully develop unconditionally stable staggered algorithms for thermoelasticity (Armero and Simo 1992, Farhat et al. 1991, Liu and Chang 1985).

For the numerical treatment of the fully coupled thermoelastic peridynamic system of equations, a staggered strategy is adopted. The system is partitioned naturally according to the structural and thermal fields; thus, the equation of motion is solved for the displacement field and the heat transfer equation is solved for the temperature field. Explicit time stepping schemes are utilized to approximate the solutions to both equations.

In order to illustrate the numerical implementation, one-dimensional peridynamic thermoelastic equations, Eq. (55), are considered, and they can be discretized in the forms

$\overline{\ddot{u}}_{(i)}^{n}=\frac{2}{\bar{\delta}^{2} \bar{A}} \sum_{j=1}^{N} \frac{\bar{\xi}_{(i)(j)}^{n}+\overline{\boldsymbol{\eta}}_{(i)(j)}^{n}}{\left|\overline{\boldsymbol{\xi}}_{(i)(j)}^{n}+\overline{\boldsymbol{\eta}}_{(i)(j)}^{n}\right|}\left(\overline{\boldsymbol{S}}_{(i)(j)}^{n}-\bar{T}_{(i)(j)}^{n}\right) \bar{V}_{(j)}$,

and

$\overline{\dot{T}}_{(i)}^{n}=\frac{2}{\bar{\delta}^{2} \bar{A}} \sum_{j=1}^{N}\left(\frac{\bar{\tau}_{(i)(j)}^{n}}{\left|\bar{\xi}_{(i)(j)}^{n}\right|}-\epsilon \frac{\overline{\dot{e}}_{(i)(j)}^{n}}{2}\right) \bar{V}_{(j)}$,

in which the term $2 /\left(\bar{\delta}^{2} \bar{A}\right)$ is assumed to be constant throughout the domain, $n$ represents the time step number, $i$ is the collocation point that is being solved for, and $j$ represents the collocation points within the horizon of $i$. The nondimensional volume of the subdomain represented by the collocation point $j$ is denoted by $\bar{V}_{(j)}$.

The discretization of a one-dimensional domain is illustrated in Fig. 2. The onedimensional domain is discretized into subdomains, with the collocation points at the center of each subdomain. 


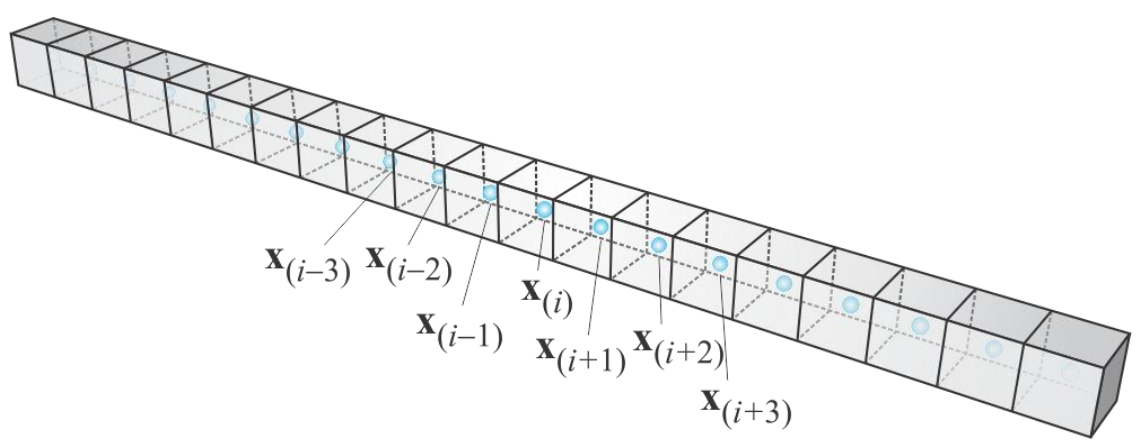

Figure 2. Discretization of one-dimensional domain with collocation points

Numerical convergence study performed by Agwai (2011) for thermal and thermomechanical analysis suggests the horizon size of $\bar{\delta}=3 \bar{\Delta}$ which is the same as the horizon size for deformation field suggested by Silling and Askari (2005). The parameter, $\bar{\Delta}$ is the nondimensional spacing between material points. The material point of interest is denoted by $i$ and it interacts with the three points to its left and right. Thus, points $j$ within the horizon of $i$ are $i-3, i-2, i-1, i+1, i+2$, and $i+3$, as shown in Fig. 2 . The nondimensional displacement, velocity, and temperature of all the collocation points are known at the $n^{\text {th }}$ time step, i.e., the current time step. Based on Fig. 2, Eq. (55a) can be discretized as

$$
\begin{aligned}
& \overline{\ddot{u}}_{(i)}^{n}=\frac{2}{\bar{\delta}^{2} \bar{A}}\left[\frac{\overline{\boldsymbol{\xi}}_{(i)(i+3)}^{n}+\overline{\boldsymbol{\eta}}_{(i)(i+3)}^{n}}{\left|\overline{\boldsymbol{\xi}}_{(i)(i+3)}^{n}+\overline{\boldsymbol{\eta}}_{(i)(i+3)}^{n}\right|}\left(\overline{\boldsymbol{s}}_{(i)(i+3)}^{n}-\bar{T}_{(i)(i+3)}^{n}\right) \bar{V}_{(i+3)}+\frac{\overline{\boldsymbol{\xi}}_{(i)(i+2)}^{n}+\overline{\boldsymbol{\eta}}_{(i)(i+2)}^{n}}{\left|\overline{\boldsymbol{\xi}}_{(i)(i+2)}^{n}+\overline{\boldsymbol{\eta}}_{(i)(i+2)}^{n}\right|}\left(\overline{\boldsymbol{s}}_{(i)(i+2)}^{n}-\bar{T}_{(i)(i+2)}^{n}\right) \bar{V}_{(i+2)}\right. \\
& +\frac{\bar{\xi}_{(i)(i+1)}^{n}+\overline{\boldsymbol{\eta}}_{(i)(i+1)}^{n} \mid}{\left|\overline{\boldsymbol{\xi}}_{(i)(i+1)}^{n}+\overline{\boldsymbol{\eta}}_{(i)(i+1)}^{n}\right|}\left(\bar{s}_{(i)(i+1)}^{n}-\bar{T}_{(i)(i+1)}^{n}\right) \bar{V}_{(i+1)}+\frac{\overline{\boldsymbol{\xi}}_{(i)(i-3)}^{n}+\overline{\boldsymbol{\eta}}_{(i)(i-3)}^{n}}{\left|\bar{\xi}_{(i)(i-3)}^{n}+\overline{\boldsymbol{\eta}}_{(i)(i-3)}^{n}\right|}\left(\bar{s}_{(i)(i-3)}^{n}-\bar{T}_{(i)(i-3)}^{n}\right) \bar{V}_{(i-3)} \\
& \left.+\frac{\overline{\boldsymbol{\xi}}_{(i)(i-2)}^{n}+\overline{\boldsymbol{\eta}}_{(i)(i-2)}^{n}}{\left|\overline{\boldsymbol{\xi}}_{(i)(i-2)}^{n}+\overline{\boldsymbol{\eta}}_{(i)(i-2)}^{n}\right|}\left(\bar{s}_{(i)(i-2)}^{n}-\bar{T}_{(i)(i-2)}^{n}\right) \bar{V}_{(i-2)}+\frac{\overline{\boldsymbol{\xi}}_{(i)(i-1)}^{n}+\overline{\boldsymbol{\eta}}_{(i)(i-1)}^{n}}{\left|\overline{\boldsymbol{\xi}}_{(i)(i-1)}^{n}+\overline{\boldsymbol{\eta}}_{(i)(i-1)}^{n}\right|}\left(\bar{s}_{(i)(i-1)}^{n}-\bar{\theta}_{(i)(i-1)}^{n}\right) \bar{V}_{(i-1)}\right]
\end{aligned}
$$

where the nondimensional stretch is denoted by $\bar{s}_{(i)(j)}^{n}$, and it is defined as

$$
\overline{\boldsymbol{s}}_{(i)(j)}^{n}=\frac{\left|\overline{\boldsymbol{\xi}}_{(i)(j)}^{n}+\overline{\boldsymbol{\eta}}_{(i)(j)}^{n}\right|-\left|\overline{\boldsymbol{\xi}}_{(i)(j)}^{n}\right|}{\left|\overline{\boldsymbol{\xi}}_{(i)(j)}^{n}\right|} .
$$

The position of the $i^{\text {th }}$ and $j^{\text {th }}$ collocation points are given by $\overline{\mathbf{x}}_{(i)}$ and $\overline{\mathbf{x}}_{(j)}$, respectively, and, as such, the nondimensional relative initial position is defined as 
$\bar{\xi}_{(i)(j)}^{n}=\overline{\mathbf{x}}_{(j)}-\overline{\mathbf{x}}_{(i)}$

The nondimensional displacements of the $i^{\text {th }}$ and $j^{\text {th }}$ collocation points are given by $\overline{\mathbf{u}}_{(i)}^{n}$ and $\overline{\mathbf{u}}_{(j)}^{n}$, respectively. Therefore, the nondimensional relative displacement becomes

$\overline{\boldsymbol{\eta}}_{(i)(j)}^{n}=\overline{\mathbf{u}}_{(j)}^{n}-\overline{\mathbf{u}}_{(i)}^{n}$,

and the term $\bar{T}_{(i)(j)}^{n}$ is defined as

$\bar{T}_{(i)(j)}^{n}=\frac{\bar{T}_{(j)}^{n}+\bar{T}_{(i)}^{n}}{2}$.

Based on Fig. 2, Eq. (55b) can be discretized as

$$
\begin{aligned}
\overline{\dot{T}}_{(i)}^{n}=\frac{2}{\bar{\delta}^{2} \bar{A}} & {\left[\left(\frac{\bar{\tau}_{(i)(i+3)}^{n}}{\left|\bar{\xi}_{(i)(i+3)}^{n}\right|}-\epsilon \frac{\overline{\dot{e}}_{(i)(i+3)}^{n}}{2}\right) \bar{V}_{(i+3)}+\left(\frac{\bar{\tau}_{(i)(i+2)}^{n}}{\left|\bar{\xi}_{(i)(i+2)}^{n}\right|}-\epsilon \frac{\overline{\dot{e}}_{(i)(i+2)}^{n}}{2}\right) \bar{V}_{(i+2)}\right.} \\
& +\left(\frac{\bar{\tau}_{(i)(i+1)}^{n}}{\left|\bar{\xi}_{(i)(i+1)}^{n}\right|}-\epsilon \frac{\overline{\dot{e}}_{(i)(i+1)}^{n}}{2}\right) \bar{V}_{(i+1)}+\left(\frac{\bar{\tau}_{(i)(i-3)}^{n}}{\left|\bar{\xi}_{(i)(i-3)}^{n}\right|}-\epsilon \frac{\overline{\dot{e}}_{(i)(i-3)}^{n}}{2}\right) \bar{V}_{(i-3)} \\
& \left.+\left(\frac{\bar{\tau}_{(i)(i-2)}^{n}}{\left|\bar{\xi}_{(i)(i-2)}^{n}\right|}-\epsilon \frac{\overline{\dot{e}}_{(i)(i-2)}^{n}}{2}\right) \bar{V}_{(i-2)}+\left(\frac{\bar{\tau}_{(i)(i-1)}^{n}}{\left|\bar{\xi}_{(i)(i-1)}^{n}\right|}-\epsilon \frac{\overline{\dot{e}}_{(i)(i-1)}^{n}}{2}\right) \bar{V}_{(i-1)}\right]
\end{aligned}
$$

where

$$
\bar{\tau}_{(i)(j)}^{n}=\bar{T}_{(j)}^{n}-\bar{T}_{(i)}^{n},
$$

and the nondimensional rate of extension between the material points is given by

$$
\overline{\dot{e}}_{(i)(j)}^{n}=\frac{\overline{\boldsymbol{\eta}}_{(i)(j)}^{n}+\overline{\boldsymbol{\xi}}_{(i)(j)}^{n}}{\left|\overline{\boldsymbol{\eta}}_{(i)(j)}^{n}+\overline{\boldsymbol{\xi}}_{(i)(j)}^{n}\right|} \cdot\left(\overline{\mathbf{u}}_{(j)}^{n}-\overline{\mathbf{u}}_{(i)}^{n}\right) .
$$

The time integration of Eq. (65) can be performed by using explicit forward and backward difference techniques. Similarly, time integration of Eq. (69) can be done by using forward difference time integration scheme. 


\section{Numerical results}

The validity of the fully coupled PD thermomechanical equations is established by constructing PD solutions to previously considered problems. The first problem is a semiinfinite bar subjected to a transient thermal boundary condition. The second problem concerns the dynamic response of a thermoelastic bar with an initial sinusoidal velocity. The solutions to these problems are obtained by constructing one-dimensional PD models. The third problem is a finite plate subjected to either a pressure shock or a thermal shock, and their combination. The solutions to these problems are obtained by constructing two-dimensional PD models. The fourth is a block of material subjected to a transient thermal boundary condition. The solution to this problem is obtained by constructing a three-dimensional PD model. During the numerical simulations, the horizon size is taken as $\bar{\delta}=3.015 \bar{\Delta}$

\subsection{A Semi-Infinite Bar Under Thermal Loading}

A semi-infinite bar is subjected to the temperature boundary condition on the bounding end. The bounding end is stress free and is gradually heated. The stress free condition on the bounding end is represented by not specifying any displacement or velocity conditions. The peridynamic discretization of the bar for thermal and deformational fields is shown in Fig. 3.

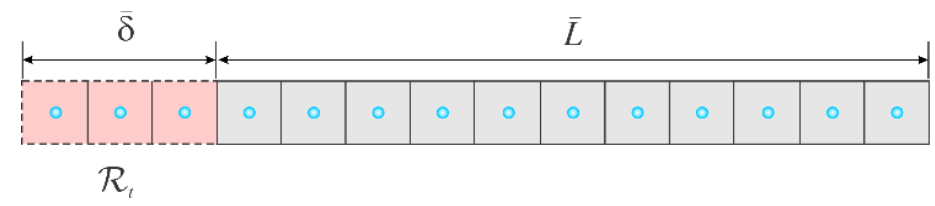

(a)

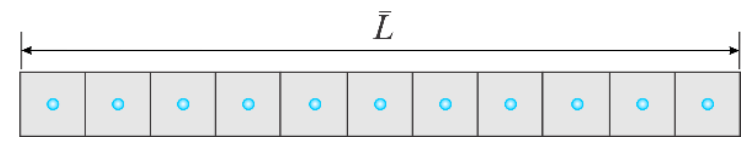

(b)

Figure 3. Peridynamic model of the fields in the one-dimensional bar: (a) thermal, (b) deformation

The peridynamic predictions for nondimensional temperature and displacement for three different coupling scenarios are compared against the classical solution reported by Nickell and Sackman (1968). The coupling coefficient values of $\epsilon=0,0.36,1$ are used to depict the decoupled, moderate, and strong coupling situations, respectively. The initial conditions for the peridynamic simulations are specified as

$\bar{u}(\bar{x}, 0)=\frac{\partial \bar{u}}{\partial \bar{t}}(\bar{x}, 0)=\bar{T}(\bar{x}, 0)=0$

The bar is subjected to temperature boundary condition as 
$\bar{T}(0, \bar{t})=\left\{\begin{array}{ll}\overline{\bar{t}} & 0 \leq \bar{t} \leq \bar{t}_{o} \\ \bar{t}_{o} & \\ 1 & \bar{t}_{o} \leq \bar{t}\end{array} \quad\right.$ with $\bar{t}_{o}=0.25$

As shown in Fig. 3, the grid spacing between the material points is $\bar{\Delta}=0.025$ with grid dimensions of $200 \times 1 \times 1$, and the time step size is $\Delta t=0.5 \times 10^{-3}$. The length of the bar is modeled as $\bar{L}=5$ with a cross sectional area of $\bar{A}=6.25 \times 10^{-4}$. The temperature boundary condition is imposed through the fictitious region, $\mathcal{R}_{t}$.

Figure 4 provides a comparison of the temperature and displacement distribution predicted by the peridynamic simulation against the finite element predictions using ANSYS at $\bar{x}=1$ for $\epsilon=0,0.36,1$. These results also agree well with those reported by Nickell and Sackman (1968). It is evident that for all three degrees of coupling the temperature at $\bar{x}=1$ increases with time in a very similar fashion while the displacement remains zero up until $\bar{t}=0.5$. At about time $\bar{t}=0.5$, the point $\bar{x}=1$ starts to be displaced in the positive direction. The effects of coupling become apparent beyond $\bar{t}=0.5$. The temperature and displacement variation for the three degrees of coupling are no longer similar. The amplitudes of the temperature and displacement decrease as the strength of the coupling is increased. The coupling accelerates the diffusion of heat as there appears to be an increase in the amount of thermal and mechanical energy dissipated. 

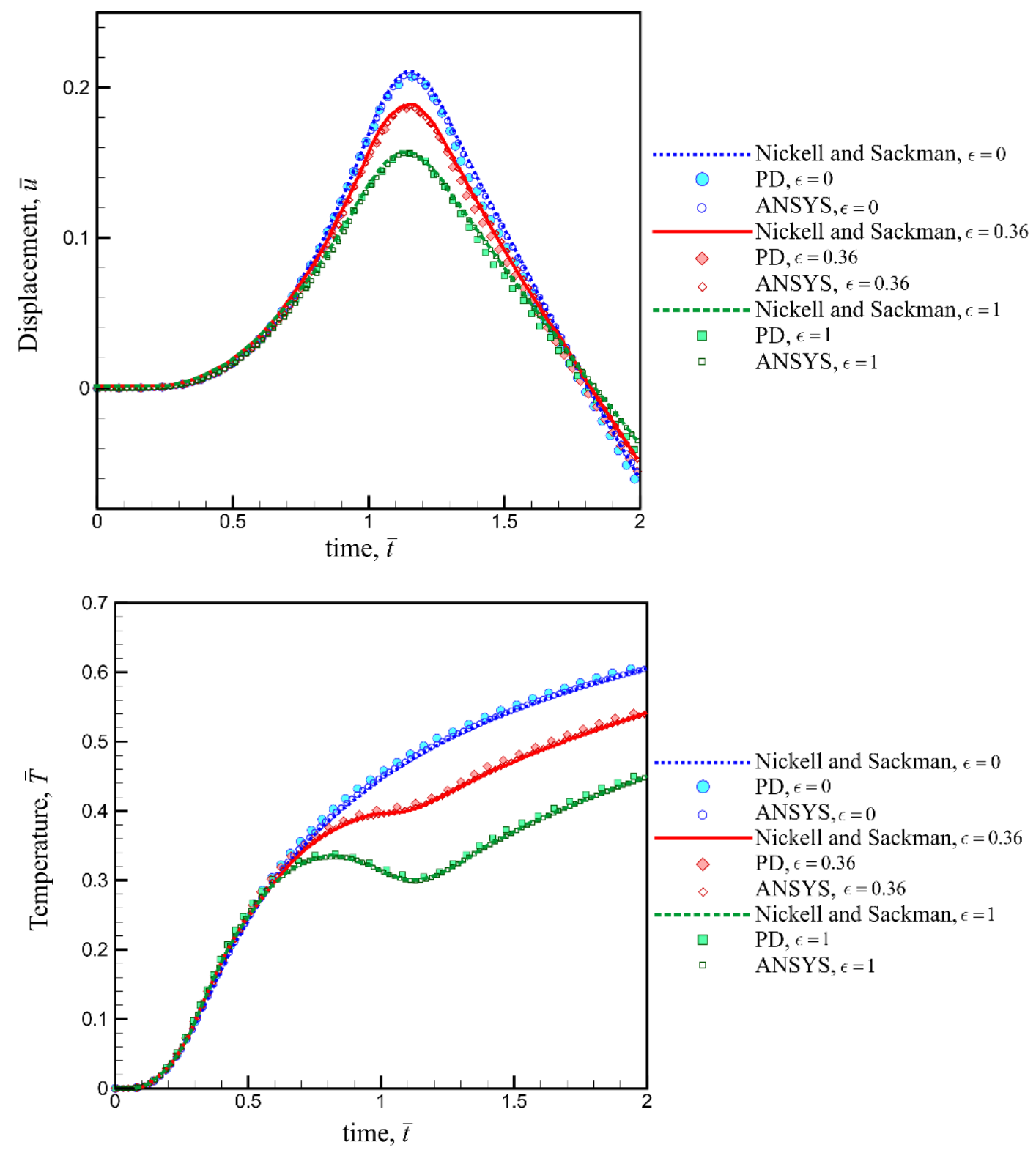

Figure 4. Displacement and temperature predictions at $\bar{x}=1$ for different coupling coefficients

\subsection{Thermoelastic Vibration of a Bar}

A bar of finite length is initially subjected to a sinusoidal velocity with zero displacement and temperature. The initial velocity is applied with a specified wave number. The ends of the bar are fixed with zero temperature and displacement. This particular thermoelastic vibration problem was considered by Armero and Simo (1992) using the finite element method. Construction of the PD solution is achieved by using the nondimensional form of 
the equations. In the peridynamic simulation, nondimensional length of the bar is $\bar{L}=100$ with a cross sectional area of $\bar{A}=4 \times 10^{-4}$. The initial conditions are specified as

$$
\begin{aligned}
& \bar{u}(\bar{x}, 0)=\bar{T}(\bar{x}, 0)=0 \\
& \frac{\partial \bar{u}}{\partial t}(\bar{x}, 0)=\sin \frac{\pi \bar{x}}{\bar{L}}
\end{aligned}
$$

The boundary conditions on the left and the right end of the bar are imposed as

$$
\begin{aligned}
& \bar{T}(0, \bar{t})=\bar{T}(\bar{L}, \bar{t})=0 \\
& \bar{u}(0, \bar{t})=\bar{u}(\bar{L}, \bar{t})=0
\end{aligned}
$$

The geometric parameters and the peridynamic discretization for the thermal and deformational fields are shown in Fig. 5 4. The grid spacing is specified as $\bar{\Delta}=0.02$ leading to dimensions of $5000 \times 1 \times 1$, and the time step size is $\Delta \bar{t}=1 \times 10^{-4}$. The temperature and displacement boundary conditions are imposed through the fictitious regions, $\mathcal{R}_{t}$ and $\mathcal{R}_{u}$, respectively.

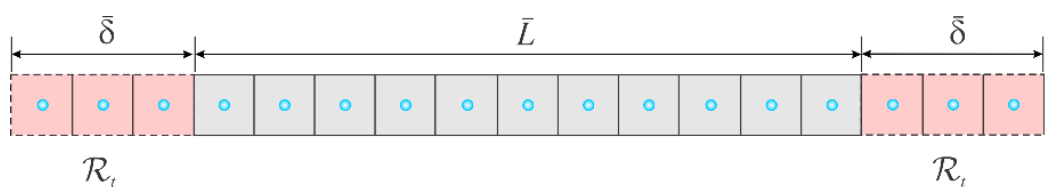

(a)

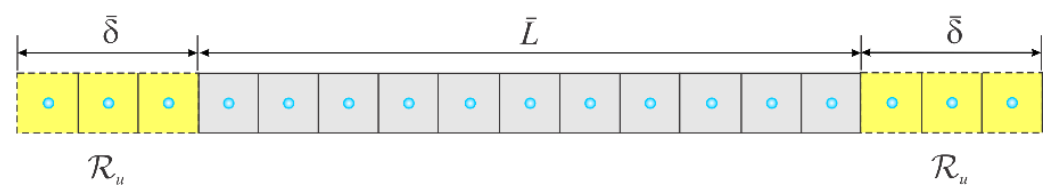

(b)

Figure 5. Peridynamic model of the fields in the one-dimensional bar: (a) thermal, (b) deformation

The resulting elastic waves are progressive traveling waves. In the case of a fully coupled thermoelastic problem, there exist two types of waves: elastic and thermal waves. Both types of waves have been modified from their uncoupled forms. The modified elastic waves are attenuated, compared to the uncoupled elastic waves, and are subjected to dispersion and damping in time. The modified thermal waves also exhibit dispersion and damping in time. The peridynamic predictions for the temporal distribution of displacement and temperature at $\bar{x}=50$ and $\bar{x}=25$, respectively, are shown in Fig. 6 for coupling coefficients of $\epsilon=0$ and $\epsilon=1$ The peridynamic predictions are also compared with the classical finite element approximations given by Armero and Simo (1992). 

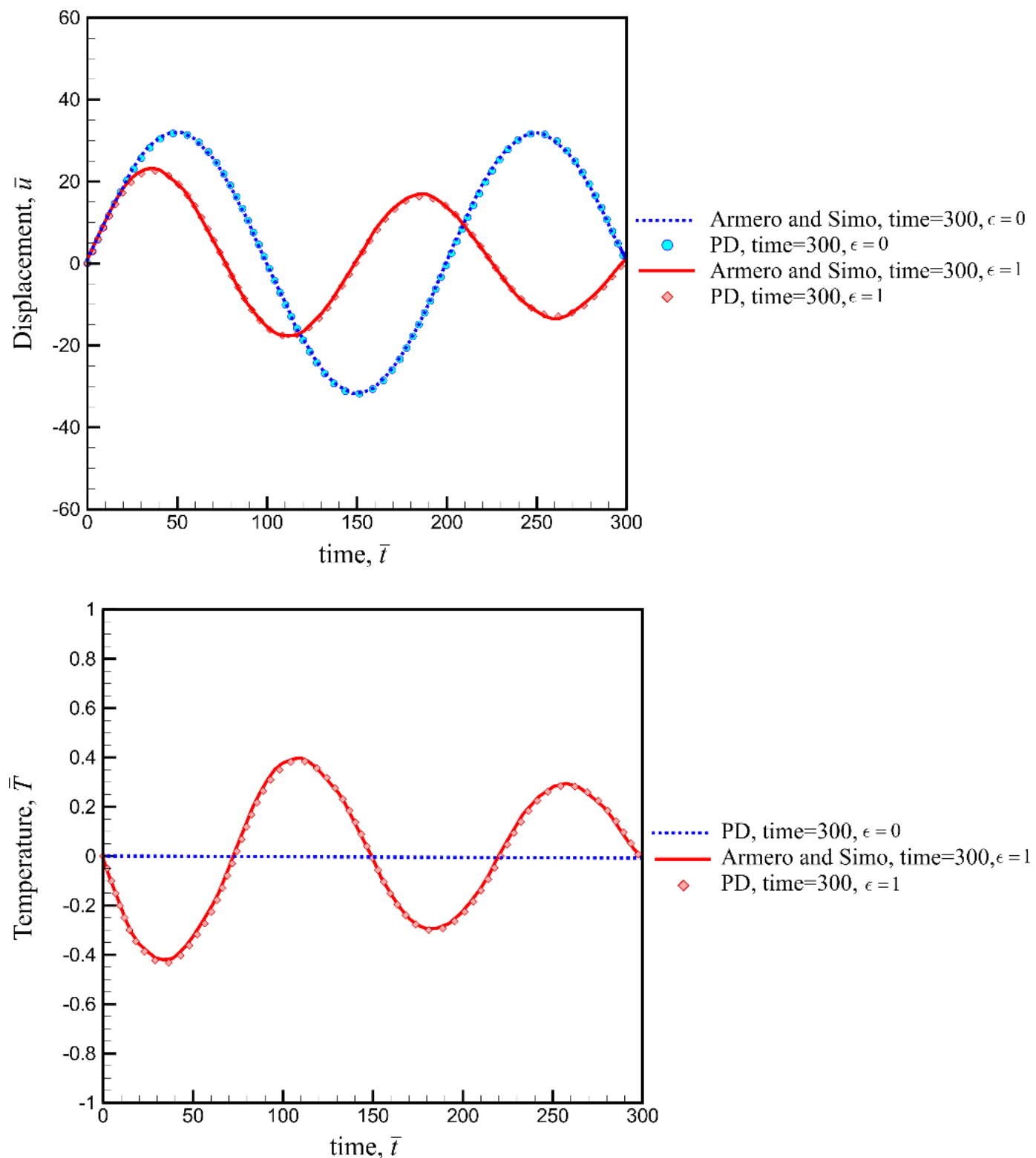

Figure 6. Displacement and temperature variation with time at $\bar{x}=50$ and $\bar{x}=25$, respectively

Although the PD and classical solutions agree well for this wave length, the PD solution captures deviates from the classical solution, and captures the nonlocal effects as the horizon approaches the order of the wavelength.

\subsection{Plate Subjected to a Shock of Pressure and Temperature, and Their Combination}

The fully coupled nondimensional PD thermomechanical equations are further verified by solving a problem previously considered by Hosseini-Tehrani and Eslami (2000) using the Boundary Element Method. It concerns a square plate of isotropic material 
under either a pressure shock or a thermal shock, and their combination on the free edge in the positive $\bar{x}$-direction. The nondimensional length and width of the square plate is 10 and it has a unit thickness. As shown in Fig. 7, it is clamped at the other edge and the insulated horizontal edges are free of any loading. The thermomechanical equations are solved for both uncoupled and coupled cases. The initial conditions are specified as

$\bar{T}(\bar{x}, \bar{y}, 0)=0$

$\bar{u}_{\bar{x}}(\bar{x}, \bar{y}, 0)=\bar{u}_{\bar{y}}(\bar{x}, \bar{y}, 0)=0$

The mechanical and thermal boundary conditions are imposed as

$\bar{T}_{, \bar{x}}(\bar{x}=10, \bar{y}, \bar{t})=0$

$\bar{T}_{, \bar{y}}(\bar{x}, \bar{y}= \pm 5, \bar{t})=0$

$\bar{u}_{\bar{x}}(\bar{x}=10, \bar{y}, \bar{t})=\bar{u}_{\bar{y}}(\bar{x}=10, \bar{y}, \bar{t})=0$

$\sigma_{\overline{y y}}(\bar{x}, \bar{y}= \pm 5, \bar{t})=\sigma_{\overline{x y}}(\bar{x}, \bar{y}= \pm 5, \bar{t})=0$

where $\bar{t}$ is the nondimensional time. The shock type loading conditions are applied as

Pressure shock

$\bar{T}(\bar{x}=0, \bar{y}, \bar{t})=0$

$\sigma_{\overline{x x}}(\bar{x}=0, \bar{y}, \bar{t})=-P(\bar{t})=-5 \bar{t} e^{-2 \bar{t}}$

Thermal shock

$\bar{T}(\bar{x}=0, \bar{y}, \bar{t})=5 \bar{t} e^{-2 \bar{t}}$

$\sigma_{\overline{x x}}(\bar{x}=0, \bar{y}, \bar{t})=0$

Combined pressure and thermal shock

$\bar{T}(\bar{x}=0, \bar{y}, \bar{t})=5 \bar{t} \exp (-2 \bar{t})$

$\sigma_{\overline{x x}}(\bar{x}=0, \bar{y}, \bar{t})=-P(\bar{t})=-5 \bar{t} e^{-2 \bar{t}}$

In the peridynamic model, a grid spacing of $\bar{\Delta}=0.05$ leads to the grid dimensions of $200 \times 200 \times 1$, and a time step of $\Delta \bar{t}=0.5 \times 10^{-3}$ is employed. 


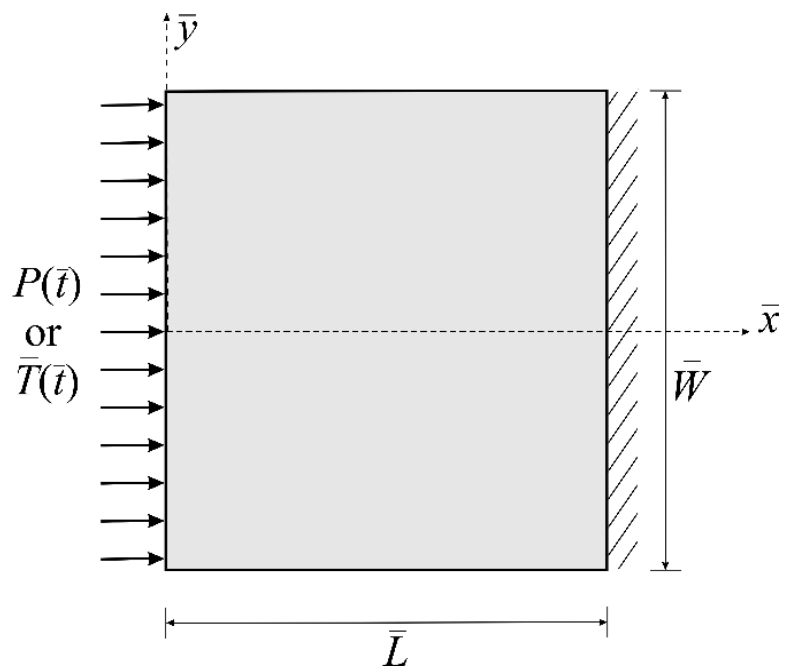

Figure 7. Geometry and boundary conditions of the plate under pressure or thermal shock

The peridynamic discretization for the thermal field is shown in Fig. 8. The temperature boundary condition is imposed in fictitious region, $\mathcal{R}_{t}$. The peridynamic discretization for the deformational field is shown in Fig. 9. The displacement boundary condition is imposed in fictitious region $\mathcal{R}_{u}$. The pressure is applied through boundary layer region $\mathcal{R}_{p}$.
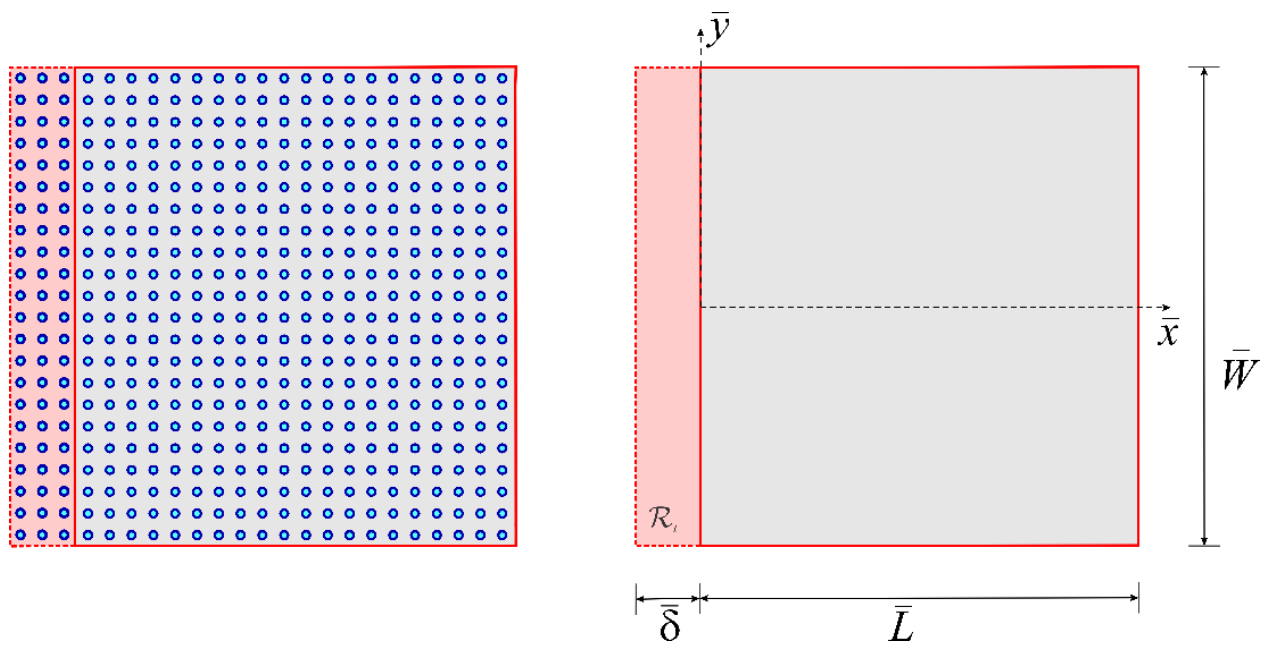

Figure 8. Peridynamic model of the thermal field in a plate 

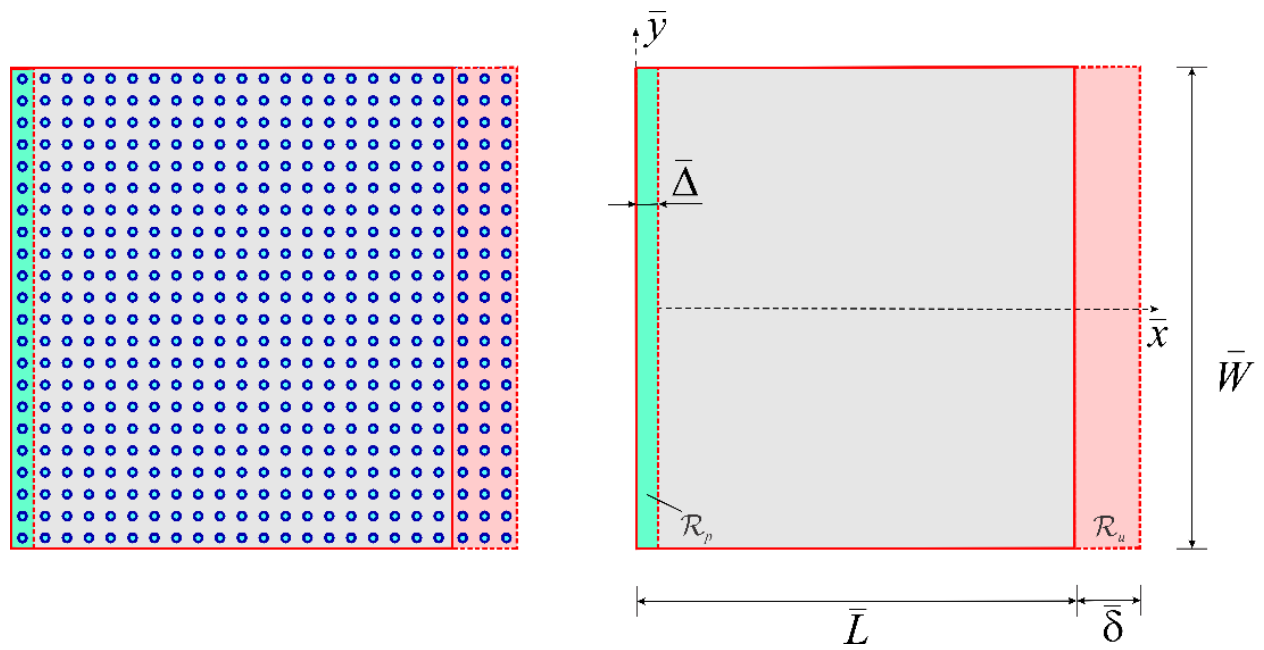

(a)
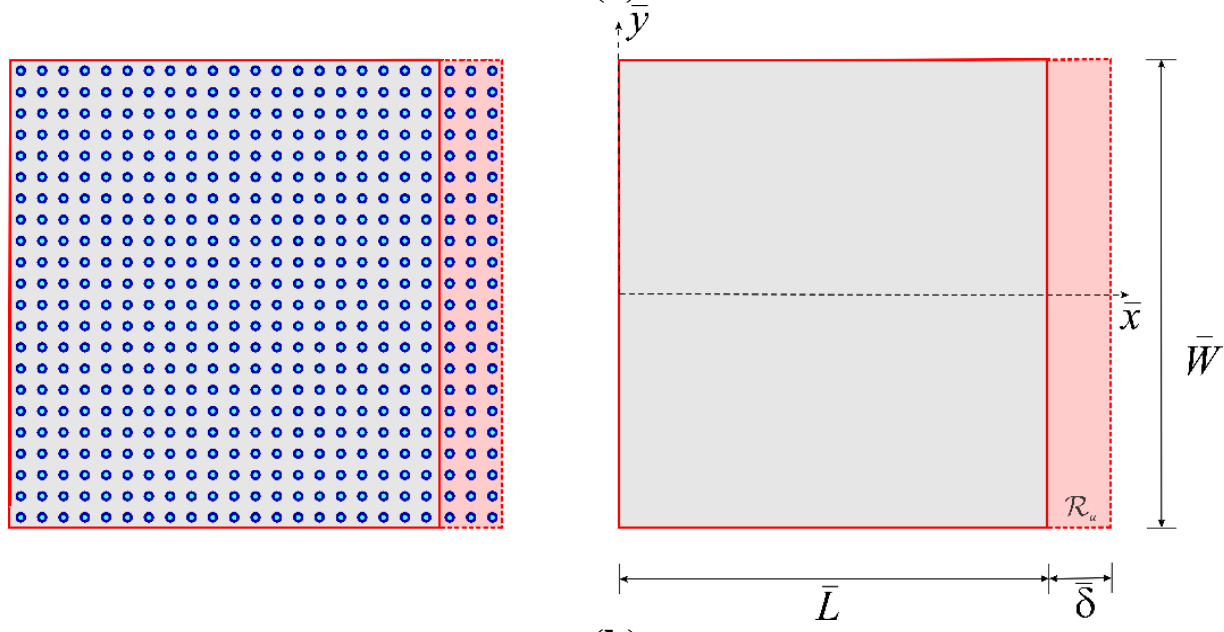

(b)

Figure 9. Peridynamic model of deformational field in a plate (a) pressure shock (b) thermal shock

Figure 10 shows the temperature and displacement variations at $\bar{y}=0$ due to the pressure shock at times $\bar{t}=3$ and $\bar{t}=6$. When the coupling coefficient is zero, no temperature change is expected. However, when the coupled effect is included, even though mechanical loading is applied, temperature change is expected. The compressive stress along the boundary causes a temperature rise. As observed in this figure, the peak of the temperature distribution moves to the right as time progresses. Figure 10 also shows the axial displacement along the $\bar{x}$-axis. The PD results are also in close agreement with the BEM results (Hosseini-Tehrani and Eslami 2000). 

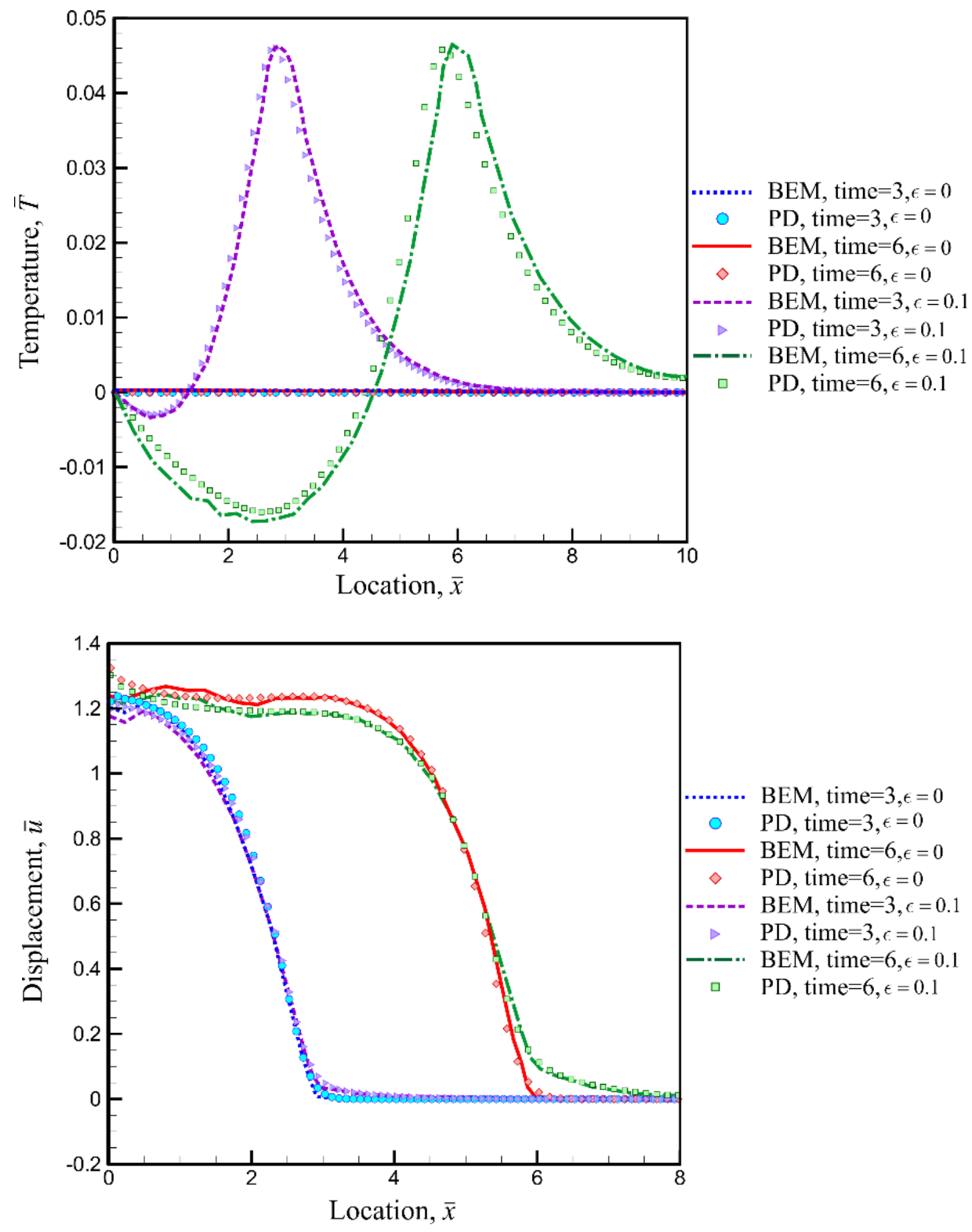

Figure 10. Temperature and displacement variations along the centerline in the plate for uncoupled $(\epsilon=0)$ and coupled $(\epsilon \neq 0)$ cases under pressure shock loading.

Figure 11 shows the temperature and displacement variations at $\bar{y}=0$ due to thermal shock at times $\bar{t}=3$ and $\bar{t}=6$. As observed, the coupling term in the thermal field causes a temperature drop, and the peridynamic predictions are in close agreement with the BEM solution published by Hosseini-Tehrani and Eslami (2000). Figure 12 shows the temperature and displacement variations at $\bar{y}=0$ due to combined pressure and thermal shock at times $\bar{t}=3$ and $\bar{t}=6$. The PD predictions are in close agreement with the BEM results by Hosseini-Tehrani and Eslami (2000). 

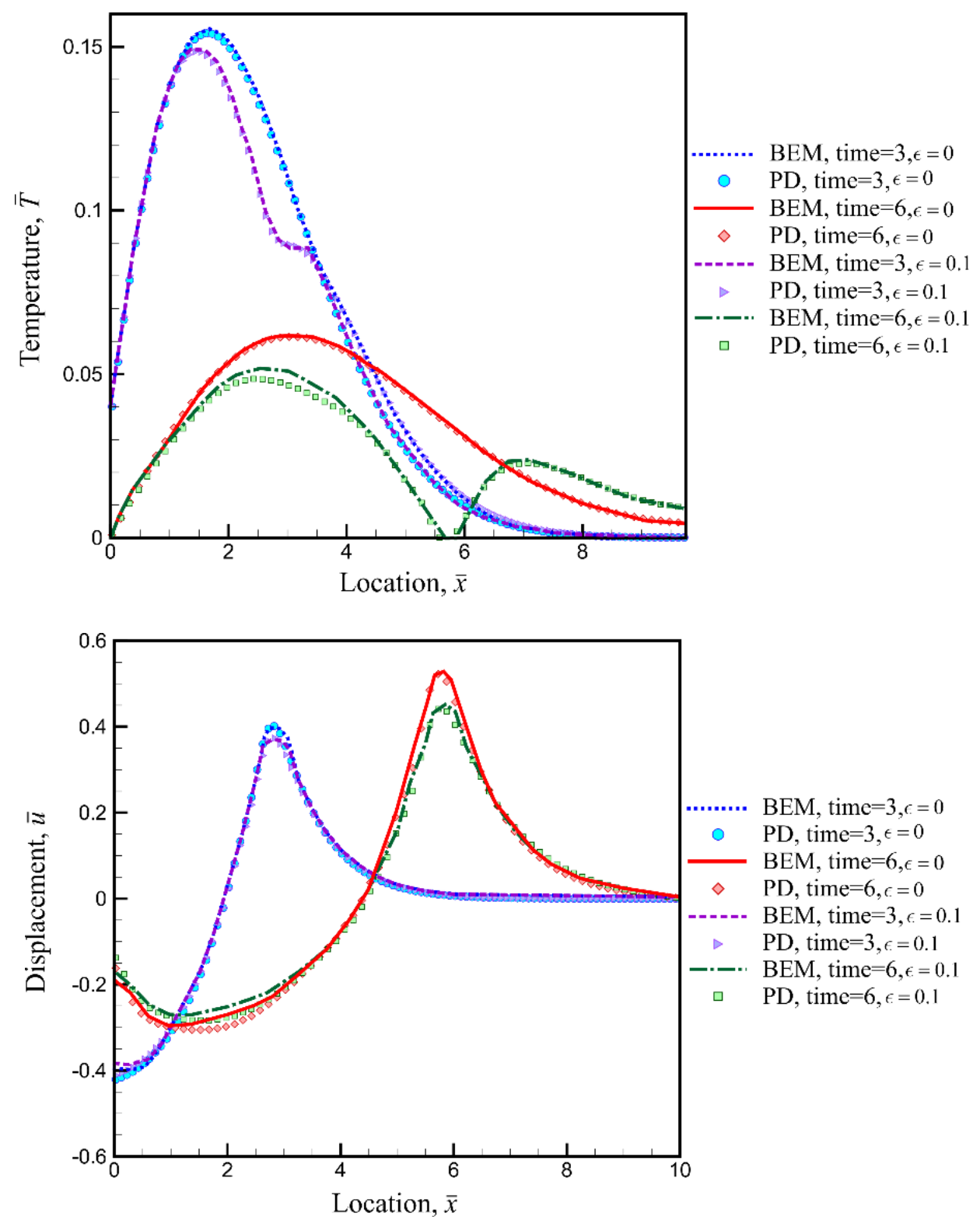

Figure 11. Temperature and displacement variations along the centerline in the plate for uncoupled $(\epsilon=0)$ and coupled $(\epsilon \neq 0)$ cases under thermal shock loading. 

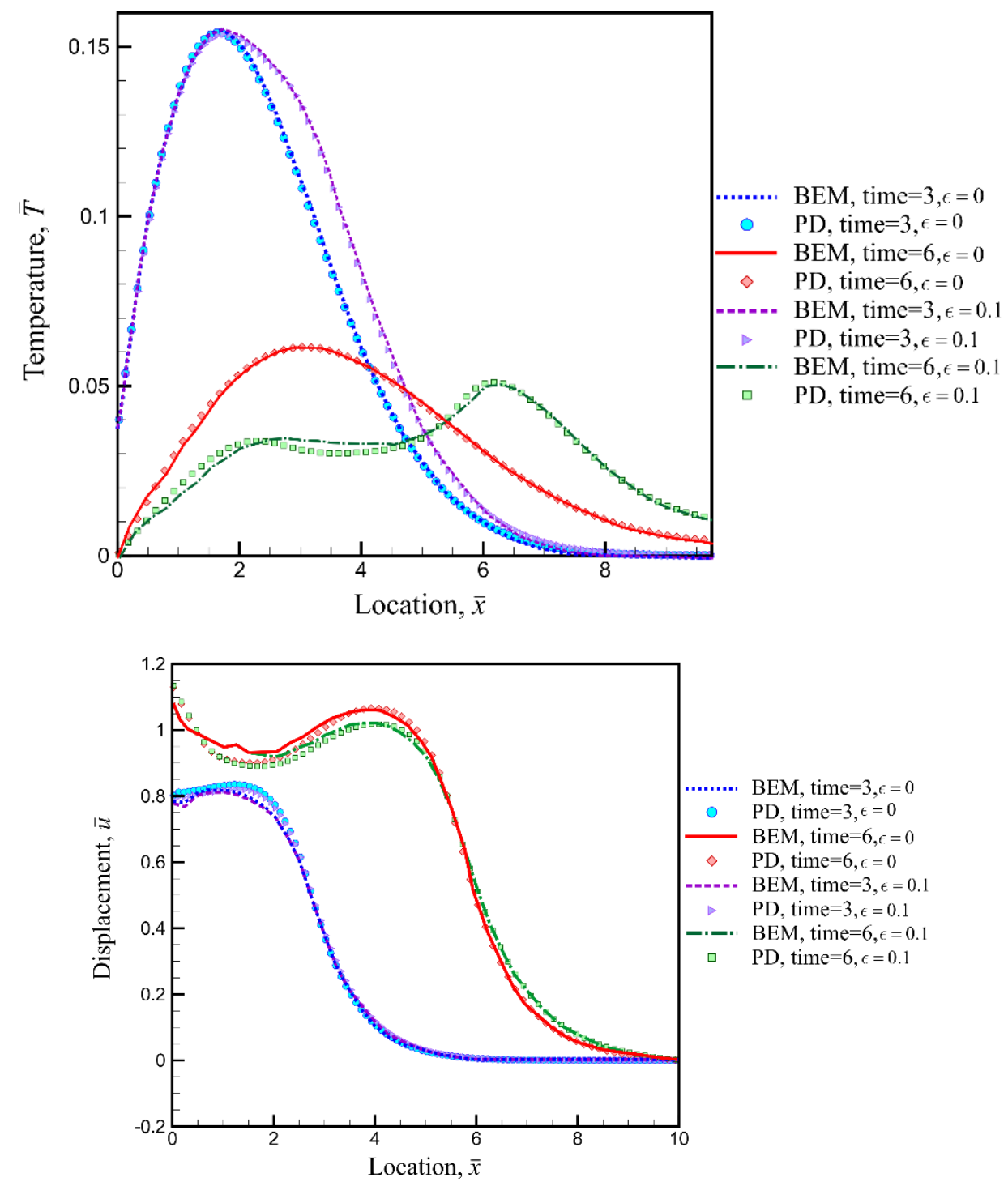

Figure 12. Temperature and displacement variations along the centerline in the plate for uncoupled $(\epsilon=0)$ and coupled $(\epsilon \neq 0)$ cases under combined thermal and pressure shock loading.

\subsection{A Block of Material Under Thermal Loading}

A three-dimensional finite block of material is gradually heated at one end, and the remaining surfaces are insulated. The nondimensional width and height of the square cross section of the block are 0.15 and it has a length of 5. As shown in Fig. 13, it is clamped at the other end without any other type of loading. The PD discretization of the thermal and deformational fields is shown in Fig. 14. 


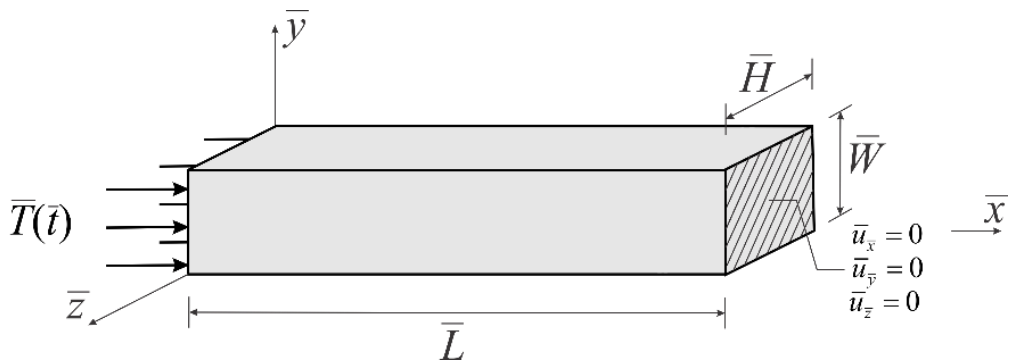

Figure 13. Geometry and boundary conditions of the block under thermal loading

The initial conditions are specified as

$\bar{u}(\bar{x}, \bar{y}, \bar{z}, 0)=\frac{\partial \bar{u}}{\partial \bar{t}}(\bar{x}, \bar{y}, \bar{z}, 0)=\bar{T}(\bar{x}, \bar{y}, \bar{z}, 0)=0$

The thermal and mechanical boundary conditions are imposed as

$\bar{T}(0, \bar{y}, \bar{z}, \bar{t}))= \begin{cases}\frac{\bar{t}}{\overline{t_{o}}} & 0 \leq \bar{t} \leq \bar{t}_{o} \\ 1 & \overline{t_{o}} \leq \bar{t}\end{cases}$

$\bar{T}_{, \bar{x}}(\bar{x}=\bar{L}, \bar{y}, \bar{z}, \bar{t})=0$

$\bar{T}_{, \bar{y}}(\bar{x}, \bar{y}=0, \bar{z}, \bar{t})=0$

$\bar{T}_{, \bar{y}}(\bar{x}, \bar{y}=\bar{W}, \bar{z}, \bar{t})=0$

$\bar{T}_{, \bar{z}}(\bar{x}, \bar{y}, \bar{z}=0, \bar{t})=0$

$\bar{T}_{\bar{z}}(\bar{x}, \bar{y}, \bar{z}=\bar{H}, \bar{t})=0$

$\bar{u}_{\bar{x}}(\bar{x}=\bar{L}, \bar{y}, \bar{z}, \bar{t})=\bar{u}_{\bar{y}}(\bar{x}=\bar{L}, \bar{y}, \bar{z}, \bar{t})=\bar{u}_{\bar{z}}(\bar{x}=\bar{L}, \bar{y}, \bar{z}, \bar{t})=0$

$\sigma_{\overline{x x}}(\bar{x}=0, \bar{y}, \bar{z}, \bar{t})=\sigma_{\overline{x y}}(\bar{x}=0, \bar{y}, \bar{z}, \bar{t})=\sigma_{\overline{x z}}(\bar{x}=0, \bar{y}, \bar{z}, \bar{t})=0$

$\sigma_{\overline{y y}}(\bar{x}, \bar{y}=0, \bar{z}, \bar{t})=\sigma_{\overline{x y}}(\bar{x}, \bar{y}=0, \bar{z}, \bar{t})=\sigma_{\overline{y z}}(\bar{x}, \bar{y}=0, \bar{z}, \bar{t})=0$

$\sigma_{\overline{y y}}(\bar{x}, \bar{y}=\bar{W}, \bar{z}, \bar{t})=\sigma_{\overline{x y}}(\bar{x}, \bar{y}=\bar{W}, \bar{z}, \bar{t})=\sigma_{\overline{y z}}(\bar{x}, \bar{y}=\bar{W}, \bar{z}, \bar{t})=0$

$\sigma_{\overline{z z}}(\bar{x}, \bar{y}, \bar{z}=0, \bar{t})=\sigma_{\overline{x z}}(\bar{x}, \bar{y}, \bar{z}=0, \bar{t})=\sigma_{\overline{y z}}(\bar{x}, \bar{y}, \bar{z}=0, \bar{t})=0$

$\sigma_{\bar{z} \bar{z}}(\bar{x}, \bar{y}, \bar{z}=\bar{H}, \bar{t})=\sigma_{\overline{x z}}(\bar{x}, \bar{y}, \bar{z}=\bar{H}, \bar{t})=\sigma_{\overline{y z}}(\bar{x}, \bar{y}, \bar{z}=\bar{H}, \bar{t})=0$

In the peridynamic model, a grid spacing of $\bar{\Delta}=0.025$ leads to the grid dimensions of $200 \times 6 \times 6$, and a time step of $\Delta \bar{t}=1 \times 10^{-4}$ is employed. 


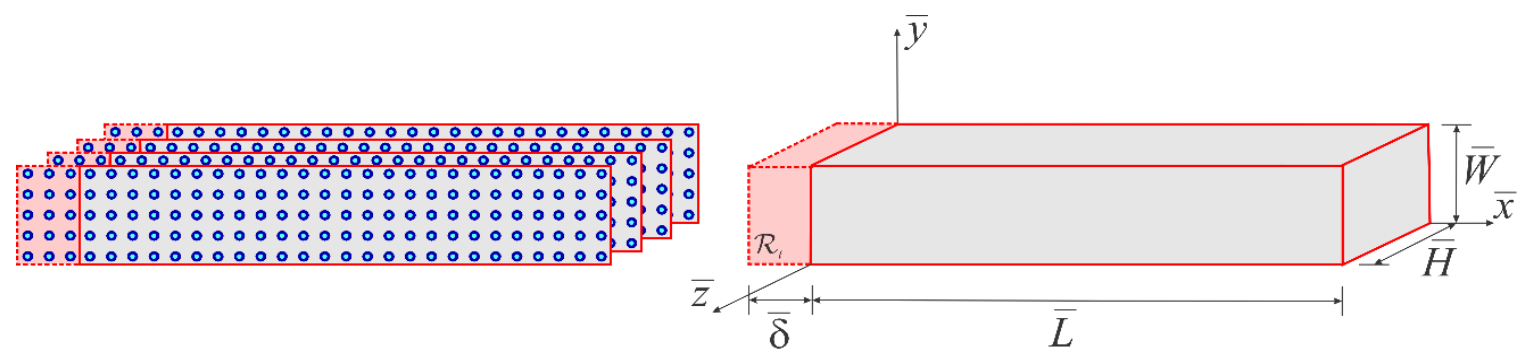

(a)

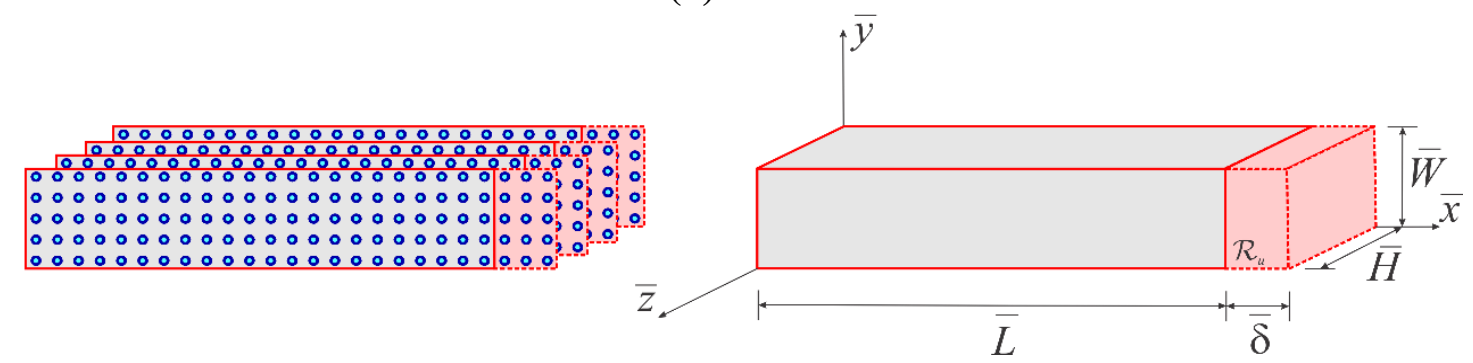

(b)

Figure 14. 3-D Peridynamic model of the fields (a) thermal, and (b) deformation

As shown in Fig. 15, the PD predictions for temperature and displacement variations along the length of the block are compared with the FEA results from ANSYS at $\bar{t}=1$ and 2 for $\epsilon=0$ and $\epsilon=1$. The comparison indicates excellent agreement. 

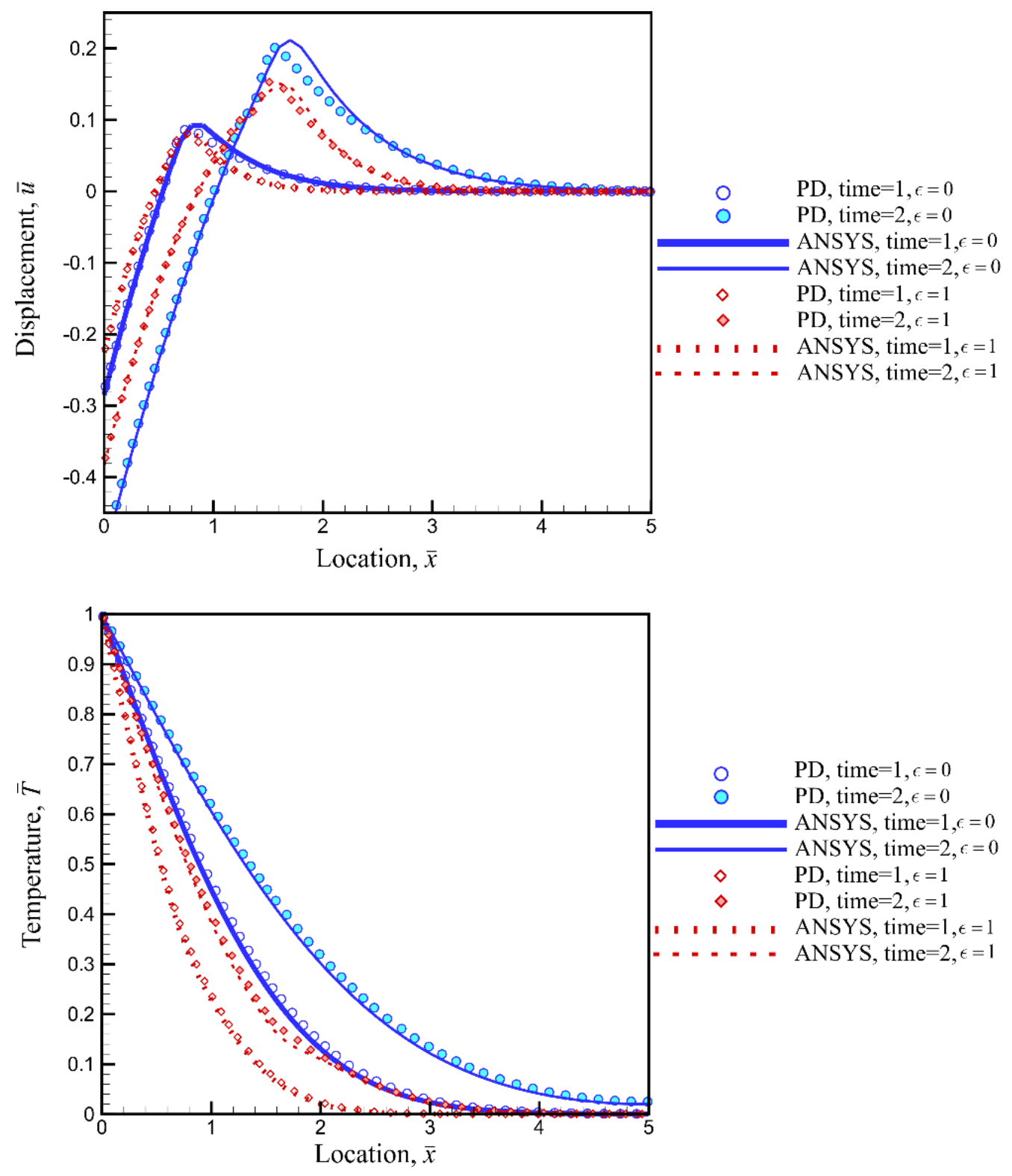

Figure 15. Displacement and temperature predictions at $\bar{y}=\bar{W} / 2, \bar{z}=\bar{H} / 2$ for coupled and uncoupled cases

\section{Conclusions}

This study describes the fully coupled structural and thermal peridynamic equations for thermomechanical analysis. The derivation of the governing equations is based upon thermodynamic considerations. The generalized state-based equations are presented along with the bond-based equations. The nondimensional form of the bond-based peridynamic thermomechanical equations leads to the coupling coefficient. A numerical scheme for solving the bond-based thermomechanical equations is described and 
peridynamic predictions are compared against classical solutions. It can be concluded that the peridynamic theory does capture the correct local thermomechanical response.

\section{References}

Agwai A (2011) A peridynamic approach for coupled fields. Dissertation, University of Arizona

Ardito R, Comi C (2009) Nonlocal thermoelastic damping in microelectromechanical resonators. J Eng Mech-ASCE 135:214-220

Armero F, Simo JC (1992) A new unconditionally stable fractional step method for nonlinear coupled thermomechanical problems. Int J Numer Meth Eng 35:737-766

Biot MA (1956) Thermoelasticity and irreversible thermodynamics. J Appl Phys 27:240253

Bobaru F, Duangpanya M (2010) The peridynamic formulation for transient heat conduction. International Journal of Heat and Mass Transfer 53: 4047-4059.

Bobaru F, Duangpanya M (2012) A peridynamic formulation for transient heat conduction in bodies with evolving discontinuities. Journal of Computational Physics 231: 2764-2785

Boley BA, Hetnarski RB (1968) Propagation of discontinuities in coupled thermoelastic problems. ASME J Appl Mech 35: 489-494

Boley BA, Tolins IS (1962) Transient coupled thermoelastic boundary value problems in the half-space. J Appl Mech 29:637-646

Brünig M, Albrecht D, Gerke S (2011) Numerical analyses of stress-triaxiality-dependent inelastic deformation behavior of aluminum alloys. Int J Damage Mech 20:299-317

Chadwick P (1960) Thermoelasticity: the dynamical theory. In: Sneddon IN, Hill R (eds), Progress in Solid Mechanics, vol. I. North-Holland, Amsterdam, pp 263-328

Chadwick P (1962) On the propagation of thermoelastic disturbances in thin plates and rods. J Mech Phys Solids 10:99-109.

Chadwick P, Sneddon IN (1958) Plane waves in an elastic solid conducting heat. J Mech Phys Solids 6:223-230

Chen TC, Weng CI (1988) Generalized coupled transient thermoelastic plane problems by laplace transform finite-element method. ASME J Appl Mech 55:377-382

Chen TC, Weng CI (1989a) Coupled transient thermoelastic response in an axisymmetric circular-cylinder by laplace transform finite-element method. Comput Struct 33:533-542

Chen TC, Weng CI (1989b) Generalized coupled transient thermoelastic problem of a square cylinder with elliptical hole by laplace transform finite-element method. $\mathbf{J}$ Therm Stresses12: 305-320

Deresiewicz H (1957) Plane waves in a thermoelastic solid. J Acoust Soc Am 29:204209

Eringen AC, Kim BS (1974a) Stress concentration at the tip of crack. Mech Res Commun $1: 233-237$

Eringen AC, Kim BS (1974b) On the problem of crack tip in nonlocal elasticity. In: Thoft-Christensen $\mathrm{P}$ (ed) Continuum mechanics aspects of geodynamics and rock fracture mechanics, proceedings of the NATO advanced study institute held in 
Reykjavik, Iceland, 11-20 August 1974. D. Reidel Publishing Co., Dordrecht, p 107113

Farhat C, Park KC, Duboispelerin Y (1991) An unconditionally stable staggered algorithm for transient finite-element analysis of coupled thermoelastic problems. Comput Methods Appl Mech Eng 85:349-365

Fung YC (1965) Foundations of solid mechanics. Prentice-Hall, Englewood Cliffs NJ

Givoli D, Rand O (1995) Dynamic thermoelastic coupling effects in a rod. AIAA J 33:776-778

Hosseini-Tehrani P, Eslami MR (2000) BEM analysis of thermal and mechanical shock in a two-dimensional finite domain considering coupled thermoelasticity. Eng Anal Bound Elem 24:249-257

Huang ZX (1999) Points of view on the nonlocal field theory and their applications to the fracture mechanics (II) - re-discuss nonlinear constitutive equations of nonlocal thermoelastic bodies. Appl Math Mech 20:764-772

Kilic B, Madenci E (2010) Peridynamic theory for thermomechanical analysis. IEEE Trans Adv Packag 33:97-105

Liu WK, Chang HG (1985) A note on numerical-analysis of dynamic coupled thermoelasticity. ASME J Appl Mech 52: 483-485

Liu WK, Zhang YF (1983) Unconditionally stable implicit explicit algorithms for coupled thermal-stress waves. Comput Struct 17: 371-374

Lychev SA, Manzhirov AV, Joubert SV (2010) Closed solutions of boundary-value problems of coupled thermoelasticity. Mech Solids 45:610-623

Macek RW, Silling SA (2007) Peridynamics via finite element analysis. Finite Elem Anal Des 43:1169-1178

Nickell RE, Sackman JL (1968) Approximate solutions in linear coupled thermoelasticity. J Appl Mech 35:255-266

Nowinski JL (1978) Theory of thermoelasticity with applications. Sijthoff \& Noordhoff International Publishers, Alphen aan den Rijn

Oden JT (1969) Finite element analysis of nonlinear problems in dynamical theory of coupled thermoelasticity. Nucl Eng Des 10:465-475

Paria G (1958) Coupling of elastic and thermal deformations. Appl Sci Res 7:463-475

Rittel D (1998) Experimental investigation of transient thermoelastic effects in dynamic fracture. Int J Solids Struct 35:2959-2973

Silling SA (2000) Reformulation of elasticity theory for discontinuities and long-range forces. J Mech Phys Solids 48:175-209

Silling SA, Epton M., Weckner O., Xu J., Askari E., (2007) Peridynamic states and constitutive modeling. Journal of Elasticity, 88: 51-184.

Silling SA, Askari E (2005) A meshfree method based on the peridynamic model of solid mechanics. Comput Struct 83:1526-1535

Silling SA (2009) Linearized theory of peridynamic states. Report SAND2009-2458. http://www.sandia.gov/ sasilli/SAND2009-2458

Silling SA, Lehoucq RB (2010) Peridynamic theory of solid mechanics. In: Aref H, van der Giessen, H (eds). Advances in applied mechanics. Elsevier, pp73-168

Soler AI, Brull MA (1965) On solution to transient coupled thermoelastic problems by perturbation techniques. J Appl Mech 32:389-399 
Stanley P (2008) Beginnings and Early Development of Thermoelastic Stress Analysis. Strain 44: 284-297

Thomson W (Lord Kelvin) (1853) On the Dynamical Theory of Heat. Transaction of the Royal Society of Edinbourgh, 20: 261-283

Ting EC, Chen HC (1982) A unified numerical approach for thermal-stress waves. Comput Struct 15:165-175

Wood WL (1990) Practical time-stepping schemes. Clarendon Press, Oxford

\section{Appendix}

Concept of State - The "state" can be viewed as a data bank to extract information about the state of material points. For example, the vector states of reference position, $\underline{\mathbf{X}}$ and deformation, $\underline{\mathbf{Y}}$ provide information about the relative position of material points in the reference and deformed configurations. The mathematical operations for such extraction of information are denoted as

$\underline{\mathbf{X}}\left\langle\mathbf{x}^{\prime}-\mathbf{x}\right\rangle=\mathbf{x}^{\prime}-\mathbf{x}$

and

$\underline{\mathbf{Y}}\left\langle\mathbf{x}^{\prime}-\mathbf{x}\right\rangle=\mathbf{y}^{\prime}-\mathbf{y}$,

in which $\mathbf{x}^{\prime}-\mathbf{x}$ and $\mathbf{y}^{\prime}-\mathbf{y}$ represent the relative position of the points $\mathbf{x}^{\prime}$ and $\mathbf{x}$ in the reference and deformed configurations. Similarly, a temperature scalar state, $\underline{\tau}$ can provide information about the temperatures, $T^{\prime}$ and $T$ at these two material points in the form

$\underline{\tau}\left\langle\mathbf{x}^{\prime}-\mathbf{x}\right\rangle=T^{\prime}-T$,

As presented by Silling et al. (2007), the dot product of two vector states, $\underline{\mathbf{A}}$ and $\underline{\mathbf{D}}$, and two scalar states, $\underline{a}$ and $\underline{d}$ can be cast as

$\underline{\mathbf{A}} \cdot \underline{\mathbf{D}}=\int_{H} \underline{\mathbf{A}}\left\langle\mathbf{x}^{\prime}-\mathbf{x}\right\rangle \cdot \underline{\mathbf{D}}\left\langle\mathbf{x}^{\prime}-\mathbf{x}\right\rangle d H$

and

$\underline{a} \cdot \underline{d}=\int_{H} \underline{a}\left\langle\mathbf{x}^{\prime}-\mathbf{x}\right\rangle \underline{d}\left\langle\mathbf{x}^{\prime}-\mathbf{x}\right\rangle d H$,

The Fréchet Derivative - Let a scalar function, $\Psi$ be dependent on a state $\underline{\mathbf{A}}$, i.e. $\Psi=\Psi(\underline{\mathbf{A}})$. Its variation is defined as 
$d \Psi=\Psi(\underline{\mathbf{A}}+d \underline{\mathbf{A}})-\Psi(\underline{\mathbf{A}})$,

in which $d \underline{\mathbf{A}}$ is the differential of $\underline{\mathbf{A}}$. Silling et al. (2007) note that if $\Psi$ is differentiable then the variation of $\Psi$ can be defined as

$d \Psi=\underline{\nabla} \Psi(\underline{\mathbf{A}}) \cdot d \underline{\mathbf{A}}$,

or

$d \Psi=\Psi, \underline{\mathbf{A}}(\underline{\mathbf{A}}) \cdot d \underline{\mathbf{A}}$,

and the term $\underline{\nabla} \Psi(\underline{\mathbf{A}})=\Psi, \underline{\underline{\mathbf{A}}}(\underline{\mathbf{A}})$ is called the Fréchet derivative of $\Psi$ at $\underline{\mathbf{A}}$. Since $\Psi$ is a scalar value function, $\Psi, \underline{\underline{\mathbf{A}}}(\underline{\mathbf{A}})$ is a state of the same order as $\underline{\mathbf{A}}$. Fréchet derivatives of various functions of states are given by Silling et al. (2007) and Silling and Lehoucq (2010).

\section{FIGURE CAPTIONS}

Fig. 1 Imposing temperature and flux constraints

Fig. 2 Discretization of one-dimensional domain with collocation points

Fig. 3 Peridynamic model of the fields in the one-dimensional bar: (a) thermal, (b) deformation

Fig. 4 Displacement and temperature predictions at $\bar{x}=1$ for different coupling coefficients

Fig. 5 Peridynamic model of the fields in the one-dimensional bar: (a) thermal, (b) deformation

Fig. 6 Displacement and temperature variation with time at $\bar{x}=50$ and $\bar{x}=25$, respectively

Fig. 7 Geometry and boundary conditions of the plate under pressure or thermal shock

Fig. 8 Peridynamic model of the thermal field in a plate

Fig. 9 Peridynamic model of deformational field in a plate: (a) pressure shock, (b) thermal shock

Fig. 10 Temperature and displacement variations along the centerline in the plate for uncoupled $(\epsilon=0)$ and coupled $(\epsilon \neq 0)$ cases under pressure shock loading.

Fig. 11 Temperature and displacement variations along the centerline in the plate for uncoupled $(\epsilon=0)$ and coupled $(\epsilon \neq 0)$ cases under thermal shock loading

Fig. 12 Temperature and displacement variations along the centerline in the plate for uncoupled $(\epsilon=0)$ and coupled $(\epsilon \neq 0)$ cases under combined thermal and pressure shock loading

Fig. 13 Geometry and boundary conditions of the block under thermal loading

Fig. 14 3-D Peridynamic model of the fields: (a) thermal, (b) deformation

Fig. 15 Displacement and temperature predictions at $(\bar{y}=\bar{W} / 2, \bar{z}=\bar{H} / 2)$ for coupled and uncoupled cases 